\author{
University of California \\ Ernest O. Lawrence \\ Radiation Laboratory
}

UCRL-7807

MASTER

\title{
THE AERODYNAMIC HEATING OF A \\ VEHICLE TRAVELING AT SUPERSONIC VELOCITIES THROUGH A COMPRESSIBLE FLUID
}

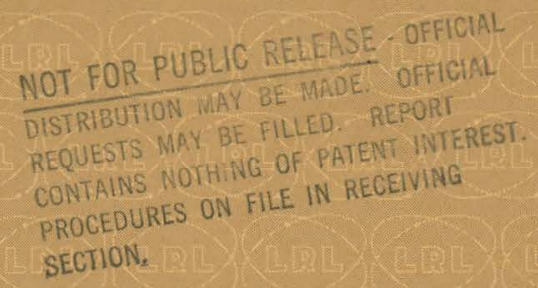

Livermore, California 


\section{DISCLAIMER}

This report was prepared as an account of work sponsored by an agency of the United States Government. Neither the United States Government nor any agency Thereof, nor any of their employees, makes any warranty, express or implied, or assumes any legal liability or responsibility for the accuracy, completeness, or usefulness of any information, apparatus, product, or process disclosed, or represents that its use would not infringe privately owned rights. Reference herein to any specific commercial product, process, or service by trade name, trademark, manufacturer, or otherwise does not necessarily constitute or imply its endorsement, recommendation, or favoring by the United States Government or any agency thereof. The views and opinions of authors expressed herein do not necessarily state or reflect those of the United States Government or any agency thereof. 


\section{DISCLAIMER}

Portions of this document may be illegible in electronic image products. Images are produced from the best available original document. 
This paper was submitted for puplication

in the open literature at for publication

prior to the is a least $A$ thonth

card. Since the U date of this Micro-

dence that it has b.A.E.C. has no evi-

per is being distributed in

form as a preprint.

Loprint.

UNIVERSITY. OF CALIFORNIA

Lawrence Radiation Laboratory

Livermore, California

Contract No. W-7405-eng-48

THE AERODYNAMIC HEA TING OF A VEHICLE TRAVELING AT SUPERSONIC VELOCITIES THROUGH A COMPRESSIBLE FLUID

Jolin Harri

July 23, 1964 
THIS PAGE

\section{WAS INTENTIONALLY LEFT BLANK}




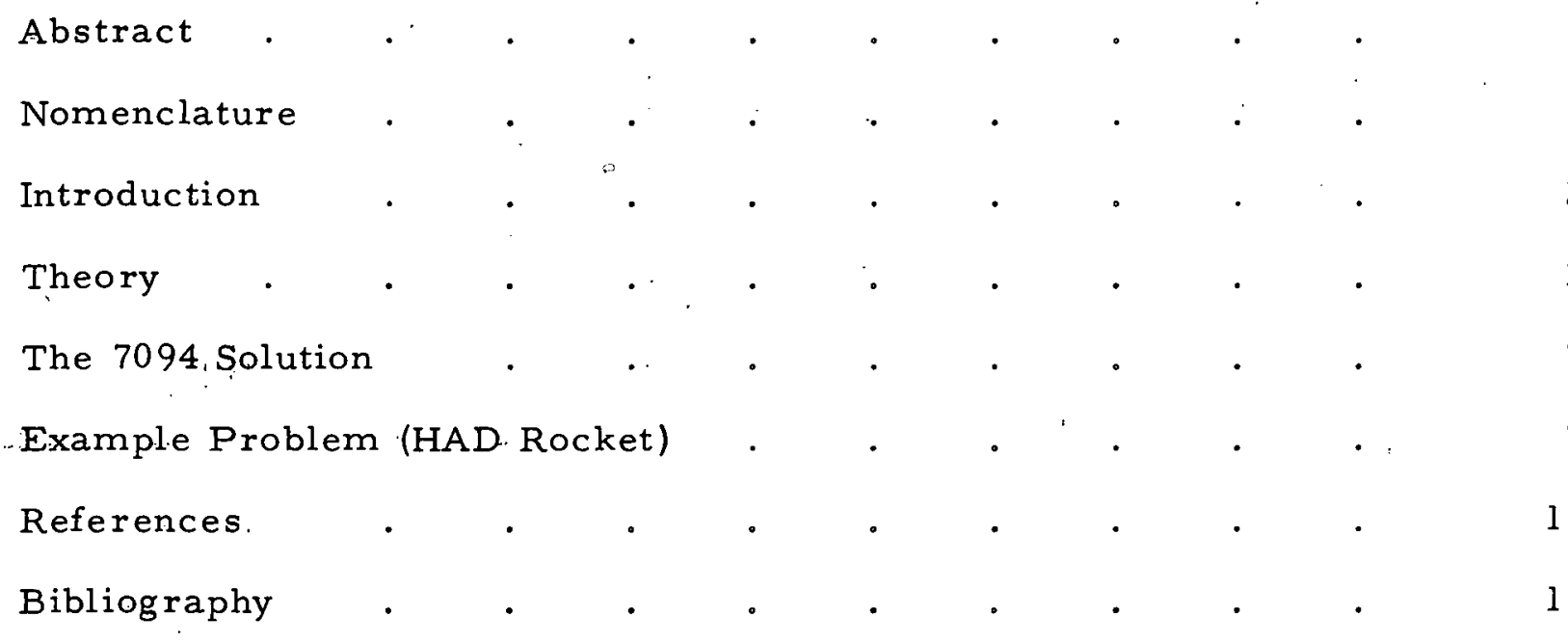

Appendices:

A FORTRAN Statements for 7094 Solution $\quad$ • . $\quad$. 12

Laminar Program $\quad . \quad \ldots \quad . \quad$. $\quad . \quad 12$

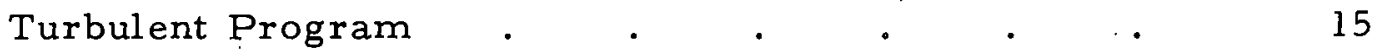

Order of Deck . . . . . . . . . . . $\quad$. $\quad$. 17

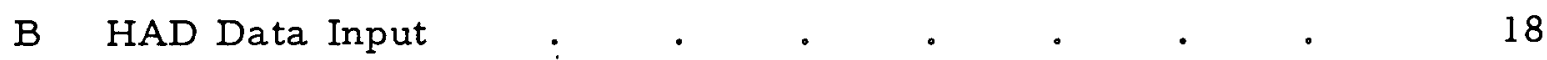

Cylindrical Section (Flat Plate Data) . . . . $\quad 18$

$30^{\circ}$ Cone Section. $\quad . \quad$. $\quad . \quad \ldots \quad . \quad 19$

$20^{\circ}$ Cone Section : $\quad . \quad \ldots \quad . \quad \ldots \quad . \quad \ldots 21$

$10^{\circ}$ Cone Section $\quad . \quad . \quad . \quad . \quad . \quad 23$

HAD Configuration Data Input . $\quad . \quad . \quad 25$

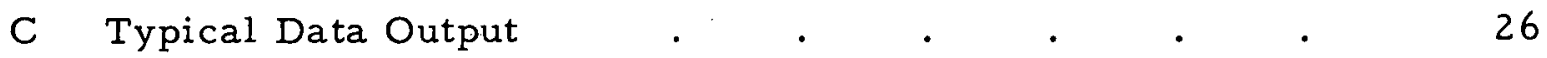

Turbulent Approximation, $\mathrm{T}_{\mathrm{W}}$ in ${ }^{\circ} \mathrm{R} \quad$. $\quad . \quad 26$

Laminar Approximation, $\mathrm{T}_{\mathrm{w}}$ in ${ }^{\circ} \mathrm{R} \quad$. . . . $\quad 33$ 
CONTENTS (Continued)

Page No.

Illustrations:

Fig. 1. Velocity profile for fluid flow along a wall . . . 38

Fig. 2. Nose cone approximated by three conical segments.

Fig. 3. Temperature distribution along HAD nose cone. Wall thickness $1 / 8$ in., time $29 \mathrm{sec}$ after launch

Fig. 4. Wall temperature 6 in. aft of nose as a function of time after launch. Wall thick-. ness $1 / 8$ in. 
THE AERODYNAMIC HEATING OF A VEHICLE TRAVELING AT SUPERSONIC VELOCITIES THROUGH A COMPRESSIBLE FLUID John Harri

Lawrence Radiation Laboratory, University of California Livermore, California

July 23, 1964

\begin{abstract}

\section{NOMENCLATURE}

$\begin{aligned} \mathrm{C}_{\mathrm{p}} & \text { Specific heat of air } \\ \mathrm{C}_{\mathrm{pw}} & \text { Specific heat of missile skin } \\ \mathrm{e} & \text { Emissivity of skin material } \\ \underset{\sim}{\mathrm{F}} & \text { External force term in Navier-Stokes equation } \\ \mathrm{G} & \text { Incident solar radiation } \\ \mathrm{h} & \quad \text { Heat transfer film coefficient } \\ \mathrm{k} & \text { Ratio of specific heats for air } \\ \mathrm{P} & \text { Pressure } \\ \mathrm{q} & \text { Heat flux } \\ \mathrm{t} & \text { Time }\end{aligned}$
\end{abstract}

An equation is derived which describes the wall temperature of a vehicle traveling at supersonic velocities through a compressible fluid. A solution of this equation is obtained by the use of the 7094 computer for both laminar and turbulent boundary layers using the Van Driest boundary layer shear stresses. Example calculations pertaining to the Lawrence Radiation Laboratory High Altitude Diagnostics rocket are included to demonstrate the use of the theory. 


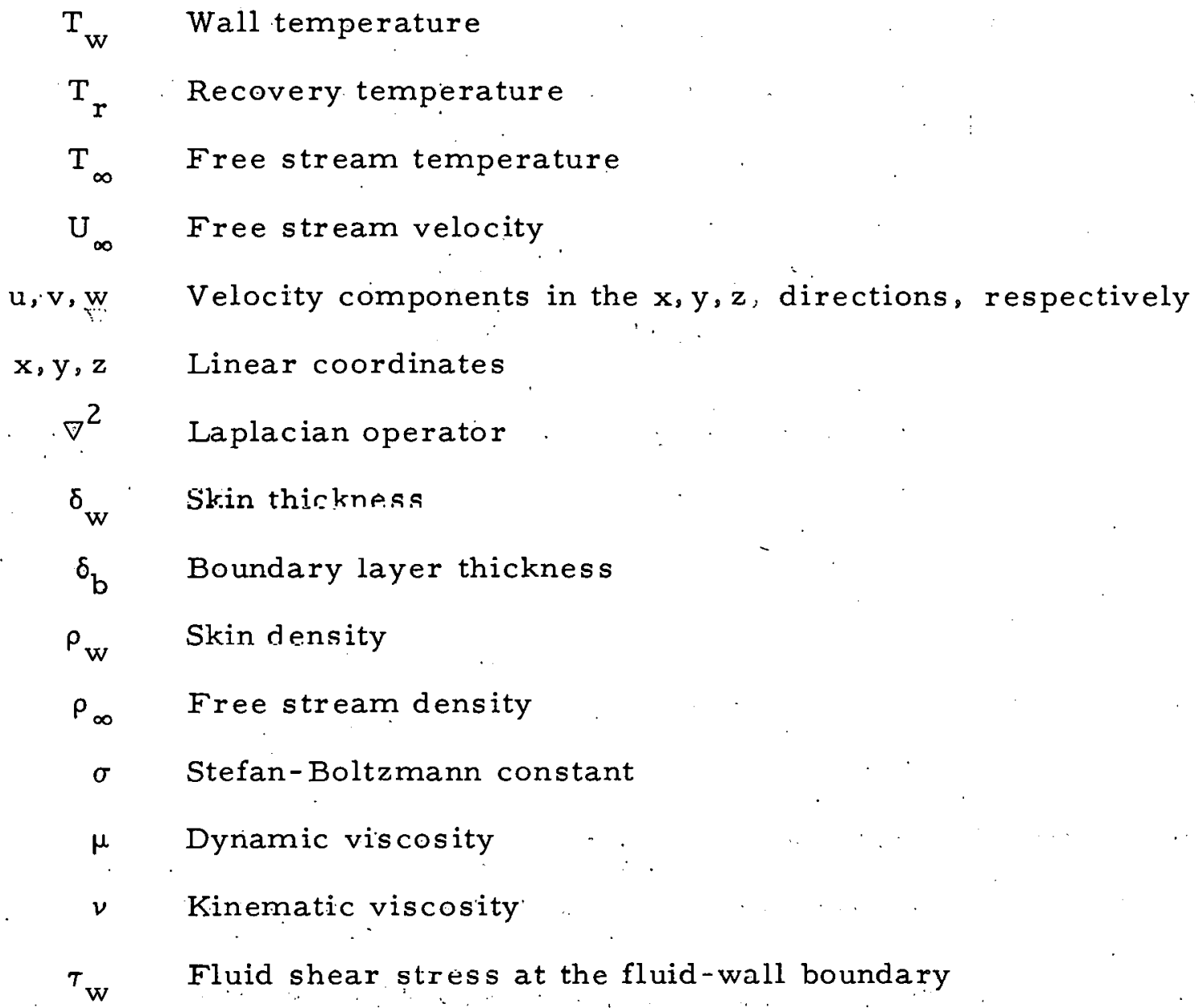

\section{INTRODUCTION}

Aerodynamic heating is a fundamental parameter in designing misisile nose cones and in selecting materials for them. Early design philosophy involved designing for the stagnation temperature, a practice which places undue concern on the heating problem. It is of interest then to examine this problem and obtain more reasonable limits for the expected temperatures, which might lead to a relaxation of the design requirements. To accomplish this goal it is necessary to examine not only the flow characteristics about the missile in question but also the nature of the boundary layer at the missile surface. 


\section{THEORY}

For the purpose of analysis, consider an increment of wall past which is flowing a compressible fluid as in Fig. $1 . \delta_{b}$ represents the boundary layer thickness, $\delta_{\mathrm{w}}$ the wall thickness, and $U_{\infty}$ the stream velocity at the edge of the boundary layer $\left(y=\delta_{b}\right)$. The velocity profile shown is typical of that through the boundary layer.

Using the following assumptions, an energy balance may be written describing the transfer of energy from the fluid to the wall.

1. Negligible heating of the material on the side of the wall opposite the fluid.

2. Negligible heat transfer in the $\mathrm{x}$ direction.

3. The temperature gradient in the y direction through the wall is small. The net heat transfer from the fluid to the wall is represented by' an increase in internal energy of the wall. This stored energy per unit area may be represented by

$$
\frac{d q}{d t}=C_{p w} \rho_{w} \delta_{w} \cdot \frac{d T_{w}}{d t}
$$

where $C_{p w}$ is the specific heat of the wall, $P_{w}$ is the density of the wall, $\delta_{w}$ is the wall thickness, $T_{w}$ is the wall temperature, and $t$ is the time.

Two mechanisms govern the heating of the wall.' First, the major energy source is the boundary layer in which the velocity gradient results in a fluid temperature increase. (The lost fluid kinetic energy now appears as fluid internal energy.) The average temperature of the boundary layer is usually termed the recovery temperature. The second mechanism is that of radiation which tends to cool the wall. The net heat transfer per unit a rea to a wall such as described in Fig. 1 which is in addition subjected to radiation from the sun is 


$$
\frac{d q}{d t}=h\left(T_{r}-T_{w}\right)-e\left(\sigma T_{w}^{4}-G\right)
$$

where $h$ is the film coefficient, $T_{r}$ is the recovery temperature, $T_{w}$ is the wall temperature, $e$ is the emissivity of the wall surface, $\sigma$ is the StefanBoltzmann constant, and $G$ is the radiation received from the sun.

Combining Eqs. (1) and (2) yields

$$
C_{p w} \rho_{w} \delta_{w} \frac{d T_{w}}{d t}=h\left(T_{r}-T_{w}\right)=e\left(\sigma T_{w}^{4}-G\right)
$$

Equation (3) now represents an energy balance per unit area in terms of the wall temperature. The crux of the problem is to determine $h$, the film coefficient. To do this the boundary layer must be examined.

The Navier-Stokes equations which describe the motion of a compressible viscous fluid are

$$
\rho \frac{\mathrm{DW}}{\mathrm{Dt}}=\underset{\sim}{\mathrm{F}}-\operatorname{grad} \mathrm{P}+\mu \nabla^{2} \underset{\sim}{W}
$$

where $\nabla^{2}$ is the Laplace operator $\frac{\partial^{2}}{\partial x^{2}}+\frac{\partial^{2}}{\partial y^{2}}+\frac{\partial^{2}}{\partial z^{2}}, \frac{D W}{D t}$ represents the total derivative of $W, W$ is the total velocity whose $x, y$, and $z$ components are $u$, $\mathrm{v}$, and $\mathrm{w}, \underset{\sim}{\mathrm{F}}$ is the resultant external force, grad $\mathrm{P}$ is the gradient of pressure, $\mu$ is the fluid viscosity, and $p$ is the fluid density. The $x$ component of (4) would be

$$
\rho\left(\frac{\partial u}{\partial t}+u \frac{\partial u}{\partial x}+v \frac{\partial u}{\partial y}+w \frac{\partial u}{\partial z}\right)=F_{x}-\frac{\partial P}{\partial x}+\mu\left(\frac{\partial^{2} u}{\partial x^{2}}+\frac{\partial^{2} u}{\partial y^{2}}+\frac{\partial^{2} u}{\partial z^{2}}\right)
$$

Considering flow only in the $\mathrm{x}$ direction, no slip between the fluid and the wall $(\mathrm{u}=.0$ for $\mathrm{y}=0) ; \mathrm{u} \approx \mathrm{U}_{\infty}$ for $\mathrm{y}=\delta_{\mathrm{b}}$. no external forces, and a velocity profile across the boundary layer which does not change appreciably with $\mathbf{x}$ or time, Eq. (5) reduces to

$$
\rho u \frac{\partial u}{\partial x}+\rho v \frac{\partial u}{\partial y}=\mu \frac{\partial^{2} u}{\partial y^{2}}
$$


From a consideration of continuity,

$$
\frac{\partial(\rho u)}{\partial x}+\frac{\partial(\rho v)}{\partial y}=0
$$

The general energy equation written with respect to the boundary layer yields

$$
\rho v \frac{\partial\left(\mathrm{C}_{\mathrm{p}} \mathrm{T}\right)}{\partial \mathrm{x}}+\rho \mathrm{v} \cdot \frac{\partial\left(\mathrm{C}_{\mathrm{p}} \mathrm{T}\right)}{\partial \mathrm{y}}=\frac{\partial}{\partial \mathrm{y}}\left(\mathrm{k} \frac{\partial \mathrm{T}}{\partial \mathrm{y}}\right)+\mu\left(\frac{\partial \mathrm{u}}{\partial \mathrm{y}}\right)^{2},
$$

where the left-hand side of Eq. (8) represents the energy influx and the righthand terms represent the energy stored and the work done by viscous shear. In Eq. (8), $\rho, C_{p}, T$, and $\mu$ are the properties of the fluid in the boundary layer.

Equations (6), (7), and (8) constitute a set of equations which represent the flow of fluid in the boundary layer. Combining these equations in the usual manner ${ }^{l}$ and recognizing the definitions of $\frac{\mathrm{dq}}{\mathrm{dt}}$ and $\tau_{\mathrm{w}}$, the shear stress in the boundary layer,

$$
\frac{d q}{d t} \doteq \frac{C_{p} \tau_{w}}{U_{\infty}}\left(T_{r}-T_{w}\right)
$$

Equation (9) represents the net energy transferred through the boundary layer in the absence of radiation. Noting Eq. (2), then

$$
\mathrm{h}=\frac{\mathrm{C}_{\mathrm{p}}{ }^{\tau} \mathrm{w}}{\mathrm{U}_{\infty}}
$$

Up to this point, no mention has been made as to whether the flow is laminar or turbulent. The equations presented are essentially for laminar flow. However, if turbulent flow is described by perturbation velocities where $U=U_{\text {laminar }}+U^{\prime}$, etc., where $U^{\prime}$ is the turbulent perturbation velocity, the resulting boundarylayer equations would be mathematically the same as Eqs. (6), (7), and (8). The solution of the resulting equations would have the same form as for the laminar case with one exception. That is, in Eq. $(10), \tau_{\mathrm{w}}$ will have different values for the laminar and turbulent cases. In 
fact, if Eq. (9) is used with the proper values of $\tau$, the error which arises in using it for both laminar and turbulent flow is consistent with the approximations made in the derivation of Eq. (9).

Van Driest ${ }^{1-3}$. has developed solutions for both laminar and turbulent flow which seem to be in reasonable agreement with experiment. Both of his $\tau_{\mathrm{w}}$ 's are developed from the momentum integral.

$$
\tau_{\mathrm{w}}=\frac{\mathrm{d}}{\mathrm{dx}} \int^{\delta_{\mathrm{b}}} \rho \mathrm{u}\left(\mathrm{U}_{\infty}-\mathrm{u}\right) \mathrm{dy}
$$

which simply states that momentum is conserved across the boundary layer. Both of Van Driest's solutions are for a flat plate and indeed some of the assumptions made in obtaining Eq. (9) were peculiar to a flat plate. Combining Eqs. (3) and (10) gives an equation which represents an energy balance on a flat plate.

$$
\mathrm{C}_{\mathrm{pw}} \delta_{\mathrm{w}} \rho_{\mathrm{w}} \frac{\mathrm{dT}}{\mathrm{dt}}=\frac{\mathrm{C}_{\mathrm{p}}{ }^{\top} \mathrm{w}}{\mathrm{U}_{\omega}}\left(\mathrm{T}_{\mathrm{r}}-\mathrm{T}_{\mathrm{w}}\right)-\mathrm{e}\left(\sigma \mathrm{T}_{\mathrm{w}}^{4}-\mathrm{G}\right) .
$$

The fact that Eq. (12) is written for a flat plate would seem to impose scvere restrictions on its use in the aerodynamic heating of a nontlat bødy. However, if the data used in the solution of Eq. (12) are properly chosen, the results are reasonably accurate. The essence of the previous statement is that the fluid conditions at $y=\delta_{b}$ and the value of $\delta_{b}$ must be taken as those which actually occur at the point on the body in question. If the flow properties about an aerodynamic body are known, the problem is one of approximating the body by a series of flat plates inclined at various angles to the flow. Inclining the flat plate to the direction of flow requires that the value of $\delta_{b}$ be adjusted accordingly. For example, the boundary layer at a given distance back from the forward edge of a cone is about $1 / 3$ as thick as the boundary layer the same distance back from the forward edge of a flat plate subjected to the same free-stream velocity. 
THE 7094 SOLUTION

Equation (12) is essentially a first-order nonlinear differential equation with nonconstant coefficients, $\tau_{\mathrm{w}}, \mathrm{U}_{\infty}$, and $\mathrm{G}$ being time dependent. The solution must be obtained numerically. Consider that the nonconstant coefficients are tabulated with respect to time. A method of solution which will yield the desired time-vs- $\mathrm{T}_{\mathrm{w}}$ data could proceed as follows:

1. Solve Eq. (12) for $\frac{d T_{w}}{d t}$.

2. Select an initial $T_{w}$ for $t=0$ 。

3. Guess $\mathrm{T}_{\mathrm{w}}$ for $\mathrm{t}=\mathrm{t}_{1}$ using some arbitrary slope.

4. Obtain from the data tables the values of $\tau_{\mathrm{w}}, \mathrm{U}_{\infty}$, and $\mathrm{G}$ for $t=t_{1}$.

5. Calculate $\frac{\mathrm{dT}_{\mathrm{w}}}{\mathrm{dt}}$ using step. 3 and step 4 information.

6. Compare 5 with the slope used in guessing $\mathrm{T}_{\mathrm{w}}$ in step 3 and choose a new $\mathrm{T}_{\mathrm{W}}$ using the slope calculated in step 5 .

7. Repeat steps 3 to 6 until $T_{\mathrm{w}}$ and the calculated slope agree. Use this new $T_{w}$ for $t=t_{1}$ and repeat the process by selecting $T_{w}$ for $t=t_{2}$, etc.

The result of the above steps will be a curve of $T_{w}$ vs $t$ which represents a solution of the differential equation. The solution scheme just described has been programmed for the IBM 7094 computer, and the details of the program appear in Appendix A.

\section{EXAMPLE PROBLEM (HAD Rocket)}

To demonstrate the use of this program, a typical problem and the results will be presented. Consider the HAD (High Altitude Diagnostics røcket) two-calibre tangent ogive nose cone. The data available is the vehicle trajectory consisting of altitude and velocity vs time. To apply the theory just developed, the tangent ogive nose cone can be approximated by a series of . : 
conical segments. (See Fig. 2.) The fluid properties over each of the conical segments must now be determined.

In the supersonic flow range the bow wave angle will be assumed to be the same as it would be if the whole nose cone had the initial cone segment angle. At each junction of the cone segments a Prandtl-Meyer expansion can be assumed. So, at point $D$ the air velocity will be nearly the same as the free stream velocity. In reality, the air is slightly overexpanded at $D$ and does not reach free stream velocity for about a calibre beyond D. However, the overexpansion is not large and will not cause an àpreciable error. Referring to the velocity-vs-time data, at each time point the following calculations must be made.

1. Determine the bow wave angle and the air properties behind the shock wave.

2. Assuming that the stagnation temperature does not change throughout the shock-expansion process, use a Prandtl-Meyer expansion with the appropriate turning angle to progress from one cone segment to the next. The required fluid properties must be calculated step by step.

3. The appropriate air data vs altitude is determined from the ARDC standard atmosphere for each time point.

The maximum time interval consistent with an integration accuracy of $\pm 1^{\circ} \mathrm{F}$ is 1 second. A typical list of time-dependent variables including the information necessary to use Van Driest's boundary layer solutions is shown in Appendix B. Also included is a list of constant data required for the solution.

The computer program uses the listed data to compute $\mathrm{T}_{\mathrm{w}}$ vs time for. eight locations specified by the values of $\mathbf{x}$ supplied in the input data. For 
the cone segments which represent the tangent ogive nose, the values of $\mathbf{x}$ - used must be modified to result in a realistic boundary layer thickness. Since the boundary layer on the conical segments is about $1 / 3$ the thickness of the boundary layer on a similar flat plate, $1 / 3$ the actual values of $\mathrm{x}$ must be used for the computation.

On the cylindrical body of the payload the appropriate length to use would be $1 / 3$ the nose length plus the distance behind the nose cone-cylindrical body junction. On the cone segment joining the cylinder of the payload to the larger cylinder of the third stage, the appropriate $x$ would be $1 / 3$ the nose length plus the cylinder length plus $1 / 3$ the distance aft of the junction between the cylindrical section of the cone segment. The values of $\mathrm{C}_{\mathrm{pw}}, \rho_{\mathrm{w}^{2}}$ and $\delta_{\mathrm{w}}$ must be the correct ones for the particular wall location.

Ëssentially, separate computer programs have been written for laminar and turbulent boundary layers. The complete solution of a given problem involves using both programs and making an estimate of the location of boundary layer transition. In general, experiment indicates ${ }^{4}$ that transition occurs at Reynolds number values of $3 \times 10^{6}$ to $8 \times 10^{6}$ along bodies of revolution as well as flat plates, so the exact location of transition cannot be accurately predicted. For uniform wall conditions (thickness, material, etc.) the maximum temperature along the wall occurs at the transition point, so it is of interest to at least make some estimate of the transition. location.

The list of data which appears in Appendix B was prepared in the manner just described, approximating the HAD nose cone by $30^{\circ}, 20^{\circ}$, and $10^{\circ}$ cone segments, and using the theoretical trajectory data obtained from the IMPACT program. ${ }^{5}$ This data was used in the 7094 solution of Eq. (12) and the results are listed in Appendix C. 
Figure 3 represents the time-temperature history of a given location on the nose cone surface. Figure 4 is a temperature profile along the payload at the time corresponding to the maximum temperature. The transition location indicated corresponds to a Reynolds number of $3 \times 10^{6}$. The dashed curve areas in Fig. 4 indicate locations at which $\delta_{\mathrm{w}}$ is very large for a short distance, thus violating some of the assumptions made in the derivation of Eq. (12).

The data shown in Figs. 3 and 4 not only are restricted by the assumptions contained in Eq. (12), but it is also as sumed that the skin surface does not become charred or insulated in any other manner. No account is taken of the possible buildup of a laminar sublayer in the turbulent boundary layer region, or of the effect on the boundary layer due to vehicle spin.

It can then be said with some degree of sureness that this data represents an upper limit for the skin temperatures. Also shown in Figs. 3 and 4 are data pertaining to $100 \%$ laminar flow. It is not unreasonable to assume that this data constitutes the lower limit of the skin temperature.

\section{Acknowledgment}

The programs in the appendices which follow were written by Mr. Ira Morrison of the Lawrence Radiation Laboratory, Livermore, Computation Center. This work was performed under the auspices of the U. S. Atomic Energy Commission. 


\section{REFERENCES}

lE. R. Van Driest, "Türbulent Boundary Layer in Compressible Fluids," J. Aeron. Sci. 18, No. 3 (March 1951).

${ }^{2} E . \cdot R$. Van Driest, "The Laminar." Boundary Layer with Variable Fluid Properties," Heat Transfer and Fluid Mechanics Institute, Berkeley, L954.

${ }^{3}$ E. R. Van Driest, "The Problem of Aerodynamic Heating," Aeron. Eng. Rev. 5, No. 10 (October 1956)。

${ }^{4}$ Carl Gazley, "Boundary Layer Stability and Transition in Subsonic and Supersonic Flow," J. Aeron. Sci. 20, No. 1 (1953).

5.. A. Hawkins, "Approximate Trajectory Equations for Unguided ThinStabilized Multistage Rockets," APGC-TN-60-27 (June 1960).

\section{BIB LIOGRA PHY}

Knudsen, J. G., and Katz, D. L., Fluid Dynamics and Heat Transfer (McGrawHill Book Company, New York, 1958).

Lee, D. B., and Faget, M. A., "Charts Adapted from Van Driest's Turbulent Flat-Plate Theory for Determining Values of Turburlent Aerodynamic Friction and Heat 'l'ransfer Coefficients," NACA-'I'N-3811, 1956.

Lee, D. B., Rumsey, C. B., and Bond, A. C. "Heat Transfer Measured in Free Flight on a Slightly Blunted $25^{\circ}$ Cone-Cylinder-Flare Configuration at Mach Numbers up to 9.89," NACA-RM-L58G21, 195.8.

Lin, C. C., Turbulent Flows and Heat Transfer (Princeton University Press, Princeton, New Jersey, 1959).

Schlichting, Hermann, Boundary Layer. Theory (McGraw-Hill Book Company, New York, 1960).

Shapiro, Ascher H., The Dynamics and Thermodynamics of Compressible Fluid Flow (two volumes) (The Ronald Press, New York, 1953). 


\section{APPENDIX A \\ FORTRAN STATEMENTS FOR 7094 SOLUTION}

Laminar Program

FORTRAN REORDER DUTPUT

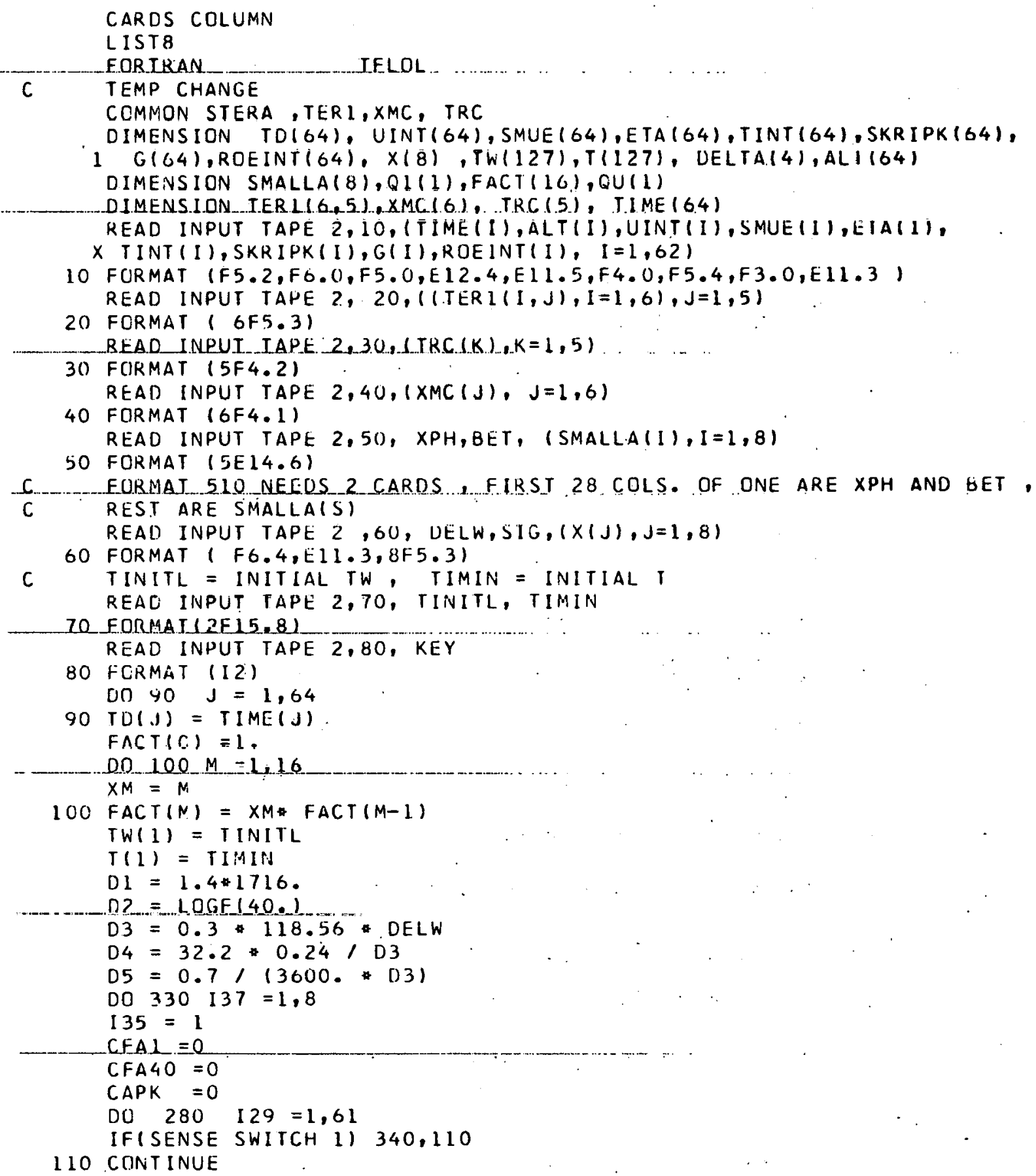


FORTRAN REORDER OUTPUT

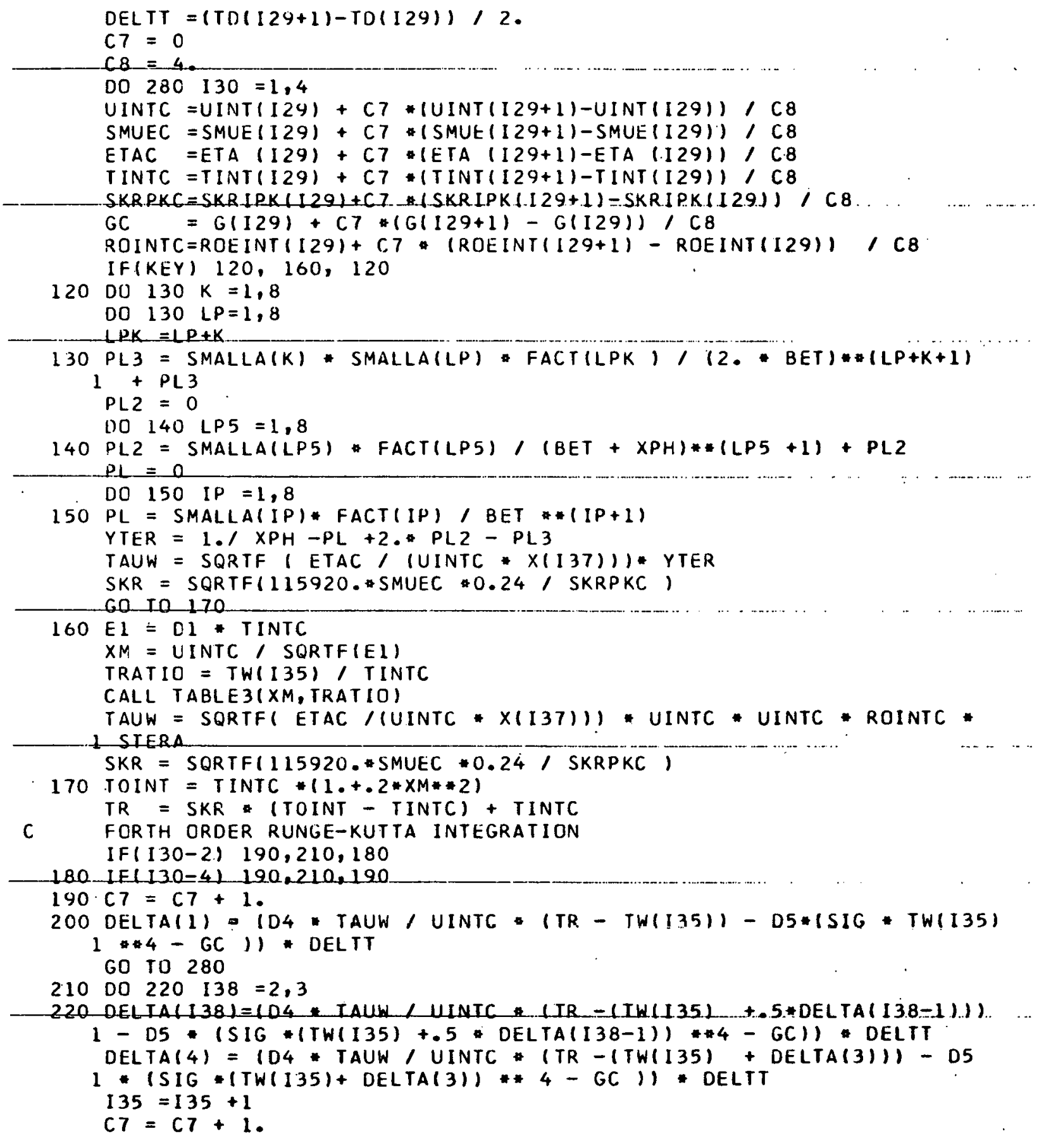


FORTRAN REDRDER OUTPUT

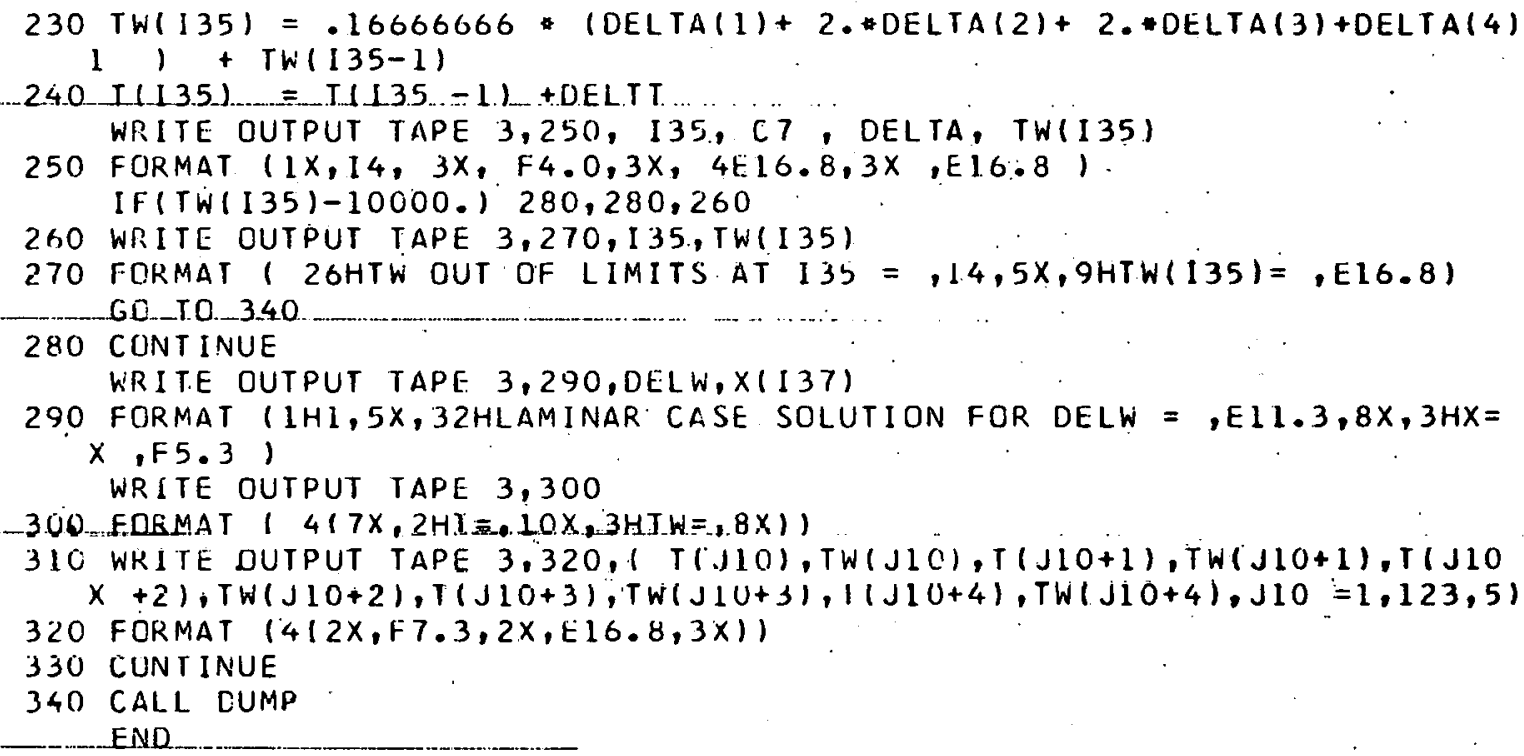




\section{Turbulent Program}

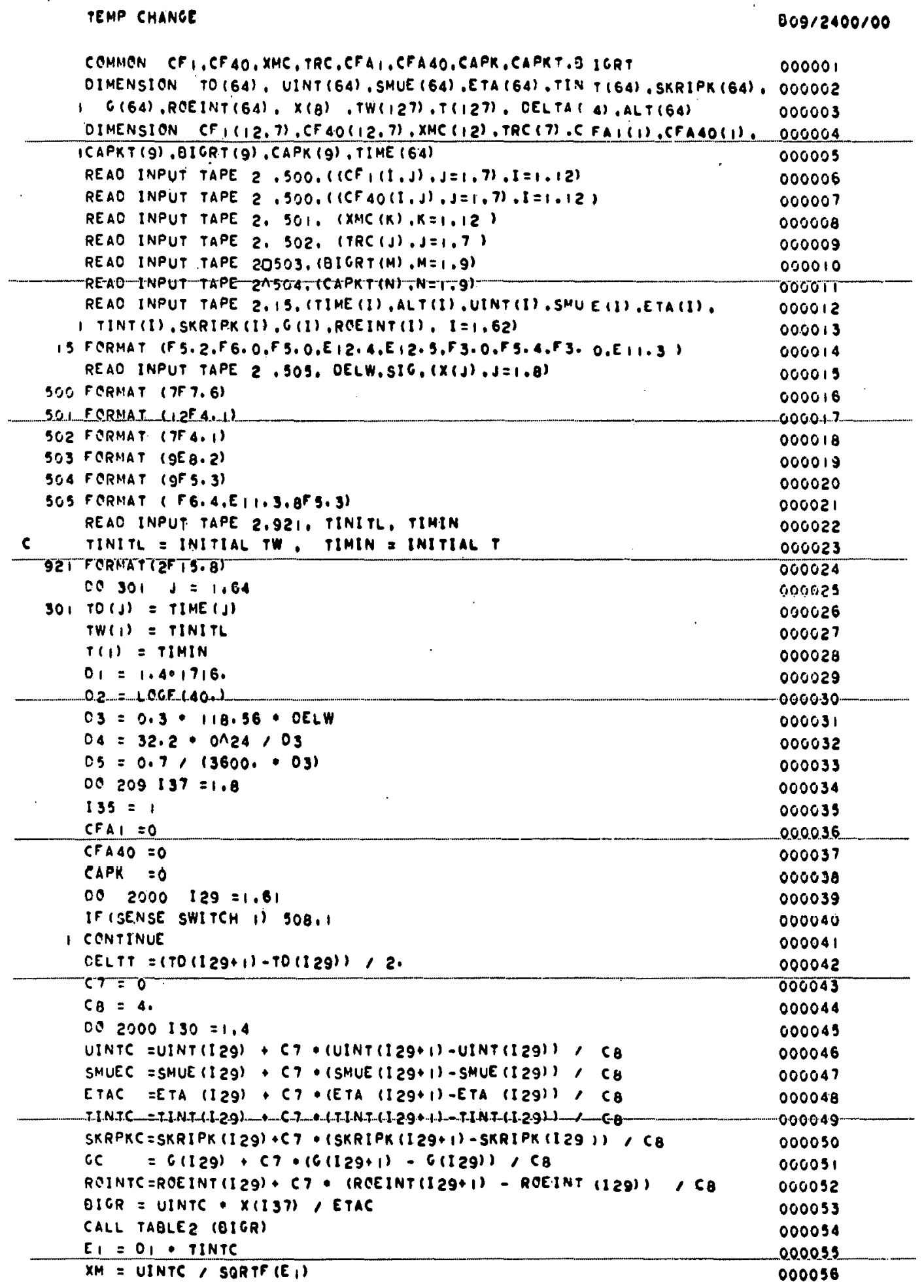




\begin{tabular}{|c|c|c|}
\hline & TEMP CHANEE & $009 / 2400 / 00$ \\
\hline & TRATIO = TW(I33), TINTC & 000057 \\
\hline . & CALL T.ABLE, IXM.TRATIO) & 000058 \\
\hline & CRAT = CFAI / CFACO & 000059 \\
\hline & $Z=$ LCCF (CRAT),$O_{2}$ & 005060 \\
\hline & $C F=C A P K-C F A T,(\theta T C R 7,1.0 E+6) \cdots 2$ & 000061 \\
\hline & :AUW $=0.3 \cdot$ ROINTC (UINTC $\cdots 2) \cdot C F$. & 000062 \\
\hline & SKR $=(113920$. SMUCC $0.24 /$ SKRPKC,$\cdots(0.33333333)$ & 000063 \\
\hline & TOINT $=$ TINTC $\cdot(1,+2 \times 2 \times M 002)$ & 000064 \\
\hline & $T R=$ SKR - (TOINT - TINTC) + TINTC & 000063 \\
\hline c & FARTH CROER RUNGE-RUTTA INTECRATION & 000066 \\
\hline & $-1 f(1-30-2)-20.3,205 \cdot .207$ & 000067 \\
\hline 207 & If $(130-4) \quad 203.203 .203$ & 000.068 \\
\hline 2,3 & $C 7=C 7+1$. & 000069 \\
\hline 2340 & 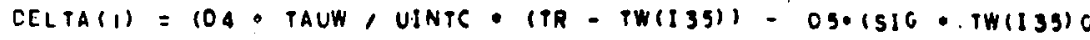 & 000070 \\
\hline & $1 \because 4-0 C 11 \cdot$ OELTT & 000071 \\
\hline & To to 2000 & $000072^{\circ}$ \\
\hline 2050 & $00.208138=2.3$ & 000073 \\
\hline $20 \mathrm{BC}$ & 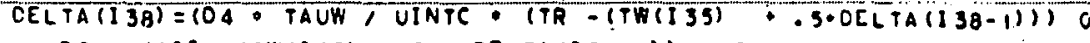 & 000074 \\
\hline & $1-$ CS $(S I C *(T W(135)+.5 \cdot$ OELTA $(138-1)) \cdot A-$ CC) $\cdot$ OELTT & 000075 \\
\hline & CELTA (4) $=104 \cdot$ TAUW, UINTC (TR- $(T W(135) \cdot$ OELTA (3)) - OS O & 000076 \\
\hline & 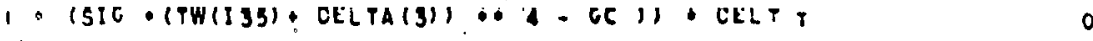 & 000077 \\
\hline & $135=135+1$ & 000078 \\
\hline & $C 7=C 7+1$. & 000079 \\
\hline 200 & 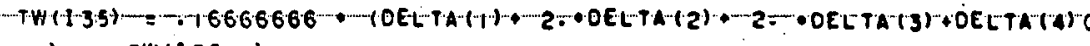 & 1000080 \\
\hline & $11+T W(135-1)$ & 000081 \\
\hline 201 & $T(135)=T(135-1)+$ OELTT & 000082 \\
\hline & WRITE CUTPUT TAPE 3,700, 135, C7, CELTA, TH(135) & 000583 \\
\hline 700 & FARMAT $(1 X, 14,3 x, F 4.0,3 x, 4 E 16,8,3 x, E 16.8)$ & 000084 \\
\hline & If (TW(135)-10000.) 2000.2000 .509 & 000085 \\
\hline 502 & WRITE CUTPUT_TAPE $3.510 .1 .35 . T W(1.3 .2)$ & -000086 \\
\hline 510 & FARMAT ( 2.6HTW OUT OF LIMITS AT I $35=.14,3 \times, 9 H T W(135)=$. E $(6.8)$ & 000087 \\
\hline & CO TO 508 & 000088 \\
\hline 2000 & CONTINUE & 000089 \\
\hline & VRITE CUTPUT TAPE $3.16 .0 E L W_{0} \times(137)$ & 000090 \\
\hline 16 & $\begin{array}{l}\text { FORNAT (IHI.3X.2 IHSOLUTIONS FER OELW =. EII.3. } 8 \times .3 H X=.59 .3) \\
\text { WIRITE CUTPUT TAPE } 3.511\end{array}$ & $\begin{array}{l}000091 \\
000092\end{array}$ \\
\hline 311 & TERMAT $(4(7 x, 2 H T=.10 X, 3 H T W=.8 X))$ & 000093 \\
\hline 210 & 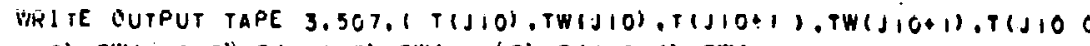 & 000094 \\
\hline & 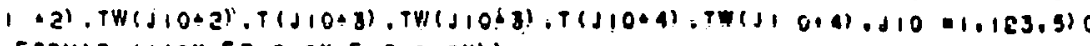 & 1000035 \\
\hline 507 & FORMAT $(4(2 x, F, 7,3,2 x, E, 16,8,3 x))$ & 000096 \\
\hline 209 & CONTINUE & 000097 \\
\hline 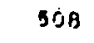 & CALL OUHP & 000098 \\
\hline
\end{tabular}




\section{Order of Deck}

\section{A. Turbulent Case}

1. I. D. card.

2. Binary deck.

3. Tables for Van Driest turbulent $\tau_{\mathrm{w}}$.

4. 62 aerodynamic data cards.

5. $\delta_{\mathrm{w}}, \dot{\sigma}$, and $\mathrm{x}$ values card.

6. Initial $\mathrm{T}_{\mathrm{w}}$ and initial $\mathrm{t}$ card.

\section{B. Laminar Case}

1. I. D. card.

2. Binary deck.

3. 62 aerodynamic data cards.

4. Tables for Van Driest laminar $\tau_{\mathrm{w}}(7$ cards).

5. Values for Harri-Appl laminar $\tau_{\mathrm{w}}$ (3 cards).

6. $\delta_{\mathrm{w}}, \sigma$, and $\mathrm{x}$ values card.

7. Initial $\mathrm{T}_{\mathrm{W}}$ and initial $\mathrm{t}$ card.

8. Key card to select 4 or 5 . 
APPENDIX B

HAD DATA INPUT

Cylindrical Section (Flat Plate Data)

\begin{tabular}{|c|c|c|c|c|c|c|c|c|}
\hline $\begin{array}{c}\text { Tluse } \\
\text { from } \\
\text { launch. } \\
\text { gec }\end{array}$ & $\begin{array}{c}\text { Altitude, } \\
\text { ft }\end{array}$ & $\begin{array}{c}\text { velocity, } \\
\text { fyeec }\end{array}$ & $\begin{array}{c}\text { Dynamic } \\
\text { viscosity } \\
10-7 \text { olugs } \\
\text { ft-gec }\end{array}$ & $\begin{array}{c}\text { Kinematic } \\
\text { vigcogity. } \\
10^{-4} \mathrm{ft}^{2} / \mathrm{sec} \\
\end{array}$ & $\begin{array}{c}\text { Air } \\
\text { temp, } \\
\text {-R }\end{array}$ & $\begin{array}{l}\text { Air thermal } \\
\text { conductivity, } \\
\text { Btu/h-ft- }{ }^{-F}\end{array}$ & $\begin{array}{l}\quad \text { Solan } \\
\text { radiation, } \\
\text { Btu } / \mathrm{h}_{-} \mathrm{ft}^{2} \\
\end{array}$ & $\begin{array}{c}\text { Air } \\
\text { density, } \\
10^{-4}{\text { slugs } / \mathrm{ft}^{3}}^{3} \\
\end{array}$ \\
\hline 2.00 & 877 & 711 & 3.719 & 1.602 & 518 & 0.01460 & 175. & 23.1 \\
\hline 3.00 & 1773 & 1100 & 3.680 & 1.641 & 512 & .01440 & 180 & 22.4 \\
\hline 4.00 & 3058 & 1498 & 3.659 & 1.681 & 508 & .01420 & 190 & 21.7 \\
\hline 4.80 & 4253 & 1792 & 3.638 & 1.723 & 503 & .01400 & 200 & 21.1 \\
\hline 5.00 & 4721 & 1769 & 3.618 & 1.766 & 500 & .01380 & 210 & 20.5 \\
\hline 6.00 & 6429 & 1648 & 3.581 & 1.840 & 495 & .01370 & 220 & 19.4 \\
\hline 7.00 & 8057 & 1609 & 3.557 & 1.903 & 490 & .01350 & 230 & 18.7 \\
\hline 8.00 & 9610 & 1535 & 3.521 & 2.002 & 485 & .01330 & 240 & 17.5 \\
\hline 9.00 & 11091 & 1166 & 3495 & 2.054 & 479 & $.01 s s 0$ & 250 & 17.0 \\
\hline 10.00 & 12418 & 1403 & 3.460 & 2.137 & 474 & .01300 & 260 & 16.5 \\
\hline 10.06 & 12505 & 1432 & 3.458 & 2.138 & 473 & .01270 & 270 & 16.0 \\
\hline 11.00 & 14154 & 1918 & 3.428 & 2.220 & 469 & .01240 & 280 & 13.5 \\
\hline 12.00 & $1630 \mathrm{~s}$ & $\angle 940^{\circ}$ & 9.909 & 3.341 & $46 !$ & 012.10 & 290 & 14.5 \\
\hline 12.87 & 18448 & $241 \%$ & 3.991 & 2.501 & 154 & . & 300 & 13.6 \\
\hline 13.00 & 18986 & 2902 & 3.326 & 2.538 & 452 & .0120 & 310 & 13.1 \\
\hline 14.00 & 21809 & 2030 & 3.262 & 2.757 & 441 & .01195 & 320 & 11.8 \\
\hline 15.00 & 24562 & 2763 & 9.201 & 3.008 & 430 & חו190 & 330 & 10.9 \\
\hline 16.00 & 27251 & 2700 & 3.153 & 3.177 & 423 & .01185 & 340 & 9.9 \\
\hline 17.00 & 29880 & 2642 & 3.090 & 3.469 & 413 & .01180 & 355 & 8.9 \\
\hline 18.00 & 32452 & 2587 & .3 .029 & 3.700 & 404 & .01175 & 370 & 8.2 \\
\hline 19.00 & 34970 & 2535 & 2.974 & 4.036 & 395 & .0113 & 380 & 7.2 \\
\hline 20.00 & 37285 & 2489 & 2.961 & 4.376 & 393 & .0110 & 390 & 6.7 \\
\hline 20.06 & 37440 & 2547 & 2.961 & 4.450 & 393 & .0110 & 100 & 6.5 \\
\hline 21.00 & 40427 & 3563 & 2.961 & 5.055 & $393^{\circ}$ & .0110 & 410 & $5.8^{\circ}$ \\
\hline 22.00 & 44512 & 4800 & 2.961 & 6.205 & 393 & .0110 & 412 & 4.7 \\
\hline EE:08 & 1003 & $6 n 5 n$ & 2941 & 7.792 & 393 & .0110 & 415 & 3.9 \\
\hline 23.00 & 49935 & 6035 & 2.961 & 8.175 & 393 & .0110 & 417 & 3.8 \\
\hline 24.00 & 55821 & 5958 & 2.961 & 13.200 & 393 & .0110 & 420 & 2.8 \\
\hline 25,00 & 61636 & 5893 & 2.961 & 14.001 & 393 & .0110 & 420 & 2.2 \\
\hline 26.00 & 67392 & 5836 & 2.961 & 16.200 & 393 & .0110 & 420 & 1.4 \\
\hline 27.00 & 73093 & 5786 & 2.961 & 21.317 & 393 & .0110 & 420 & 0.95 \\
\hline 28.00 & 78748 & 5741 & 2.961 & 30.390 & 393 & .0110 & 420 & .91 \\
\hline 2.9 .00 & 83501 & 5700 & 2.961 & 34.390 & 393 & .0110 & 420 & .85 \\
\hline $30 . n n$ & 88400 & 5660 & 2.961 & 54.350 & 393 & .0117 & $4 \angle U$ & 35 \\
\hline $3.1,0 u$ & 94030 & 5620 & 2.362 & 74350 & 400 & .01185 & 420 & .41 \\
\hline 32.00 & 98950 & 5580 & 2.961 & 85.456 & 402 & .0120 & 420 & .33 \\
\hline 33,00 & 104400 & 5540 & 2.961 & 113.4 & 406 & .0122 & 420 & .26 \\
\hline 34.00 & 109650 & 5500 & 3.090 . & 157.2 & 413 & .0124 & 420 & .21 \\
\hline 35.00 & 114850 & 5460 & 3.240 & 220.5 & 424 & .0127 & 420 & .17 \\
\hline 36.00 & 120400 & 5440 & 3.339 & 287.6 & 439 & .0130 & 420 & .11 \\
\hline 37.00 & 126540 & $5+20$ & 3.510 & 498.9 & 460 & .0133 & 420 & .08 \\
\hline 38.00 & 133178 & 5386 & 3.590 & 570.5 & 493 & .0137 & 420 & .07 \\
\hline 39.00 & 136500 & 5350 & 3.809 & 690.5 & 510 & .0141 & 420 & .05 \\
\hline 40.00 & 142100 & 5320 & 3.850 & 827.9 & 535 & .0145 & 420 & .04 \\
\hline 41.00 & 147020 & 5290 & 3.880 & 1200 & 557 & .0150 & 420 & .035 \\
\hline 42.00 & 152100 & 5260 & 4.032 & 133?? & 585 & .0158 & 420 & .03 \\
\hline 43.00 & 157500 & 5230 & 4.101 & 1790 & 605 & .0165 & 420 & .025 \\
\hline 44.00 & 163010 & 5200 & 4.332 & 2050 & 618 & .0168 & 420 & .02 \\
\hline 45.00 & 168250 & 5170 & 4.332 & 2886 & 627 & .0171 & 420 & .015 \\
\hline 46.00 & 174000 & 5140 & 4.332 & 3050 & 630 & .0173 & 420 & .010 \\
\hline 47.00 & 180300 & 5109 & 4.332 & 3886 & 630 & .0173 & 420 & .0091 \\
\hline 48.00 & 184287 & 5072 & 4.332 & 4230 & 630 & .0173 & 420 & .0085 \\
\hline 49.00 & 189100 & 5040 & 4.332 & 5232 & 630 & .0173 & 420 & .008 \\
\hline 50.00 & 193900 & 5010 & 4.332 & 5405 & 630 & .0173 & 420 & .0078 \\
\hline 51.00 & 199100 & 4980 & 4.227 & 6844 & 621 & .0173 & 420 & .0074 \\
\hline 52.00 & 203500 & 4950 & 4.177 & 6850 & 607 & .0170 & 420 & .006 \\
\hline 53.00 & 207810 & 4920 & 4.099 & 7010 & 593 & .0167 & 420 & .0053 \\
\hline 54.00 & 212600 & 4890 & 4.080 & 8467 & 575 & 0163 & 420 & .0048 \\
\hline 55.00 & 217500 & 4860 & 4.001 & 10600 & 558 & .0160 & 420 & .0043 \\
\hline 56.00 & 222500 & 4830 & 3.916 & 11300 & 543 & .0157 & 420 & .0037 \\
\hline 57.00 & 227500 & 4800 & 3.727 & 13000 & 526 & .0153 & 420 & .003 \\
\hline 58.00 & 232223 & 4765 & 3.695 & 13400 & 510 & 0.0150 & 420 & 0.002 \\
\hline
\end{tabular}


HAD Data Input for $30^{\circ}$ Cone Section

All the previous data remains the same except velocity, temperature, and density, whose new values are as listed below.

\begin{tabular}{|c|c|c|c|c|}
\hline $\begin{array}{c}\text { Time, } \\
\text { sec } \\
\end{array}$ & $\begin{array}{c}\text { Velocity, } \\
\mathrm{ft} / \mathrm{sec}\end{array}$ & $\begin{array}{c}\text { Temperature, } \\
{ }^{\circ} \mathrm{R} \\
\end{array}$ & $10^{-4}$ & $\begin{array}{l}\text { ensity, } \\
\text { slugs } / \mathrm{ft}^{3} \\
\end{array}$ \\
\hline 2.00 & 711 & 562 & & 24.4 \\
\hline \multirow[t]{2}{*}{3.00} & 1100 & 612 & & 27.9 \\
\hline & (Normal shock) & & & \\
\hline 4.00 & 840 & 619 & . & 34.7 \\
\hline 4.80 & 1057 & 649 & . & 39.3 \\
\hline 5.00 & 1031 & 643 & & 40.0 \\
\hline 6.00 & 941 & 636 & & 36.4 \\
\hline 7.00 & 816 & 633 & & 33.0 \\
\hline 8.00 & 778 & 611 & & 30.0 \\
\hline 9.00 & 806 & 588 & & 27.7 \\
\hline 10.00 & 856 & 569 & & 30.1 \\
\hline 10.06 & 834 & 573 & & 32.0 \\
\hline 11.00 & 1179 & 622 & & 34.5 \\
\hline 12.00 & 1541 & 671 & & 37.0 \\
\hline 12.87 & 1785 & 733 & & 45.2 \\
\hline 13.00 & 1780 & 728 & & 43.5 \\
\hline 14.00 & 1755 & 689 & & 37.3 \\
\hline 15.00 & 1710 & 680 & & 34.5 \\
\hline 16.00 & 1672 & 666 & & 30.6 \\
\hline 17.00 & 1645 & 641 & & 28.0 \\
\hline 18.00 & 1610 & 625 & & 26.0 \\
\hline 19.00 & 1580. & 608 & & 24.1 \\
\hline 20.00 & 1550 & 602 & & 19.8 \\
\hline 20.06 & 1605 & 604 & & 26.5 \\
\hline 21.00 & 2050 & 775 & & 34.9 \\
\hline 22.00 & 2540 & 965 & & 44.3 \\
\hline 22.87 & 3010 & 1120 & & 53.1 \\
\hline 23.00 & 3000 & 1121 & & 48.0 \\
\hline
\end{tabular}




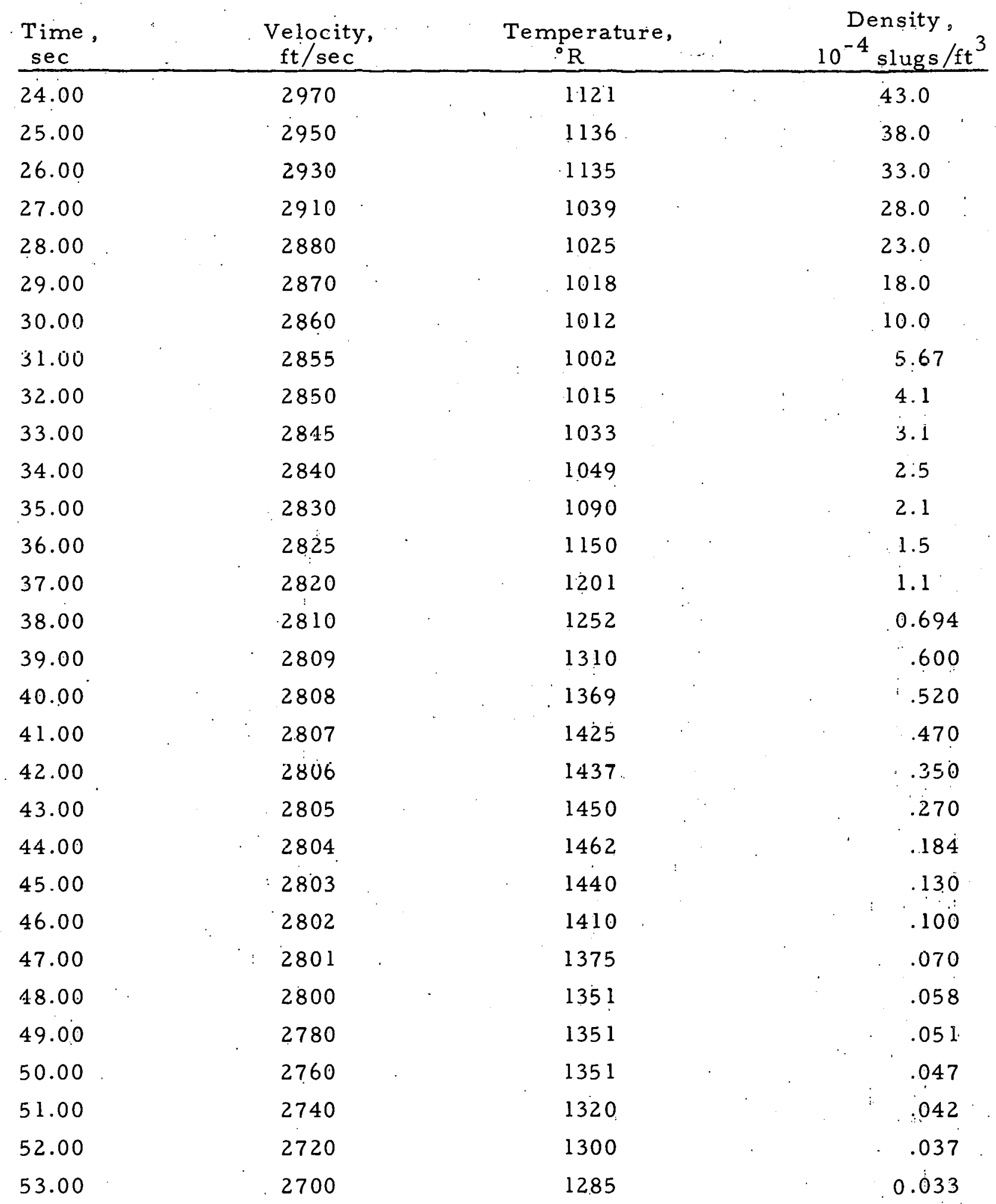




\begin{tabular}{cccc}
$\begin{array}{c}\text { Time } \\
\text { sec }\end{array}$ & $\begin{array}{c}\text { Velocity } \\
\mathrm{ft} / \mathrm{sec}\end{array}$ & $\begin{array}{c}\text { Temperature, } \\
{ }^{\mathrm{R}}\end{array}$ & $\begin{array}{c}\text { Density, } \\
10^{-4} \mathrm{slugs}_{\mathrm{ft}}{ }^{3}\end{array}$ \\
\hline 54.00 & 2680 & 1275 & 0.028 \\
55.00 & 2660 & 1250 & .024 \\
56.00 & 2640 & 1225 & .016 \\
57.00 & 2620 & 1200 & .015 \\
58.00 & 2590 & 1170 & 0.0145 \\
\hline
\end{tabular}

HAD Data Input for $20^{\circ}$ Cone Section

All the previous data remained the same except velocity, temperature, and density., whose new values are as listed below.

\begin{tabular}{|c|c|c|c|c|}
\hline $\begin{array}{l}\text { Time, } \\
\text { sec }\end{array}$ & $\begin{array}{c}\text { Velocity, } \\
\mathrm{ft} / \mathrm{sec}\end{array}$ & $\begin{array}{c}\text { Temperature, } \\
{ }_{\mathrm{R}}\end{array}$ & . & $10^{-\frac{\text { Denșity }}{4} \text { slugs } / \mathrm{ft}^{3}}$ \\
\hline 2.00 & 711 & 562 & & 23.8 \\
\hline \multirow[t]{2}{*}{3.00} & 1100 & 612 & & 26.2 \\
\hline & (Normal shock) & & $\therefore$ & \\
\hline 4.00 & 920 & 578 & & 29.9 \\
\hline 4.80 & 1395 & 584 & & 30.1 \\
\hline 5.00 & 1360 & 578 & . & 30.7 \\
\hline 6.00 & 1305 & 568 & & 27.5 \\
\hline 7.00 & 1230 & 562 & & 26.0 \\
\hline 8.00 & 1155 & 552 & 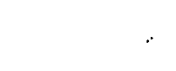 & 24.8 \\
\hline 9.00 & 1090 & 544 & & 23.5 \\
\hline 10.00 & 1043 & 535 & & 24.0 \\
\hline 10.06 & 10.57 & 535 & & 24.3 \\
\hline 11.00 & 1500 & 555 & & 24.9 \\
\hline 12.00 & 1910 & 577 & & 35.8 \\
\hline 12.87 & 2240 & 605 & & 28.2 \\
\hline 13.00 & 2228 & 602 & & $27: 2$ \\
\hline 14.00 & 2175 & 584 & & 23.8 \\
\hline 15.00 & 2120 & 568 & & 22.0 \\
\hline 16.00 & 2085 & 556 & & 19.5 \\
\hline 17.00 & 2040 & 536 & & 18.0 \\
\hline
\end{tabular}




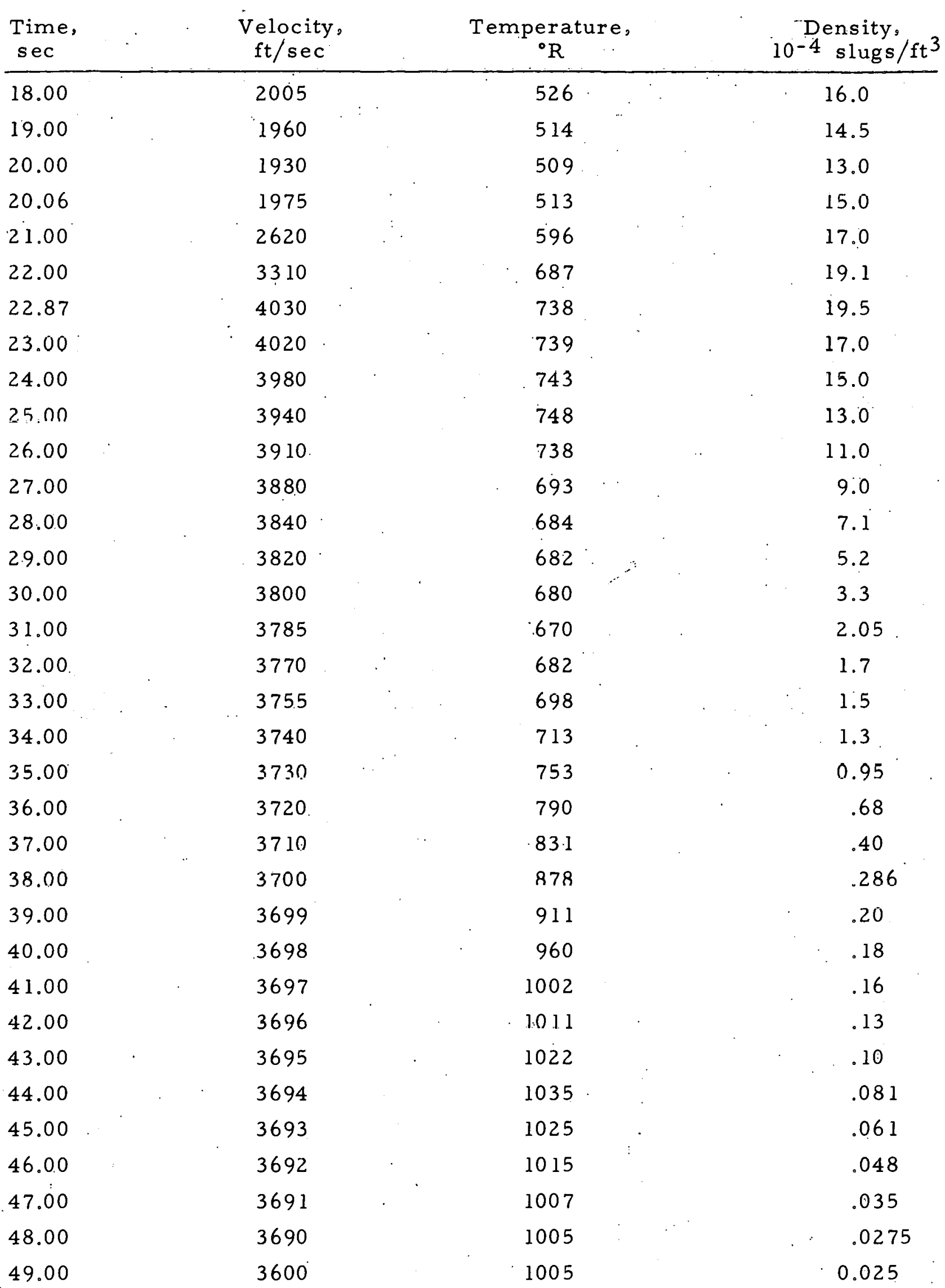




\begin{tabular}{cccc}
$\begin{array}{c}\text { Time, } \\
\text { sec }\end{array}$ & $\begin{array}{c}\text { Velocity, } \\
\text { ft/sec }\end{array}$ & $\begin{array}{c}\text { Temperature, } \\
{ }^{\circ} \text { R }\end{array}$ & $\begin{array}{c}\text { Density, } \\
10^{-4} \text { slugs } / \mathrm{ft}^{3}\end{array}$ \\
\hline 50.00 & 3520 & 1005 & 0.023 \\
51.00 & 3480 & 993 & .021 \\
52.00 & 3460 & 970 & .019 \\
53.00 & 3440 & .959 & .017 \\
54.00 & 3420 & 943 & .015 \\
55.00 & 3400 & 921 & .013 \\
56.00 & 3380 & 902 & .011 \\
57.00 & 3380 & 883 & .009 \\
58.00 & 3388 & 868 & 0.0069 \\
\hline
\end{tabular}

\section{HAD Data Input for $10^{\circ}$ Cone Section}

All the previous data remains the same except velocity; temperature, and density, whose new values are as listed below.

\begin{tabular}{|c|c|c|c|}
\hline $\begin{array}{l}\text { Time } \\
\text { sec }\end{array}$ & $\begin{array}{c}\text { Velocity } \\
\mathrm{ft} / \mathrm{sec}\end{array}$ & $\begin{array}{c}\text { Temperature } \\
{ }^{\circ} \mathrm{R}\end{array}$ & $\begin{array}{l}\text { Density, } \\
10-4 \text { slugs } / \mathrm{ft}^{3}\end{array}$ \\
\hline 2.00 & 711 & 562 & 23.1 \\
\hline 3.00 & 1100 & 612 & 23.8 \\
\hline 4.00 & 1350 & 535 & 24.7 \\
\hline 4.80 & 1650 & 520 & 23.7 \\
\hline 5.00 & 1615 & 529 & 24.4 \\
\hline 6.00 & 1545 & 522. & 22.2 \\
\hline 7.00 & 1480 & 513 & 21.0 \\
\hline 8.00 & 1405 & 499 & 20.0 \\
\hline 9.00 & 1335 & 500 & 19.1 \\
\hline 10.00 & 1276 & 496 & 18.9 \\
\hline 10.06 & 1294 & 498 & 18.7 \\
\hline 11.00 & 1760 & 502 & 18.5 \\
\hline 12.00 & 2228 & 506 & 18.3 \\
\hline 12.87 & 2645 & 510 & 18.2 \\
\hline 13.00 & 2635 & 507 & 17.6 \\
\hline 14.00 & 2560 & 495 & 15.8 \\
\hline
\end{tabular}




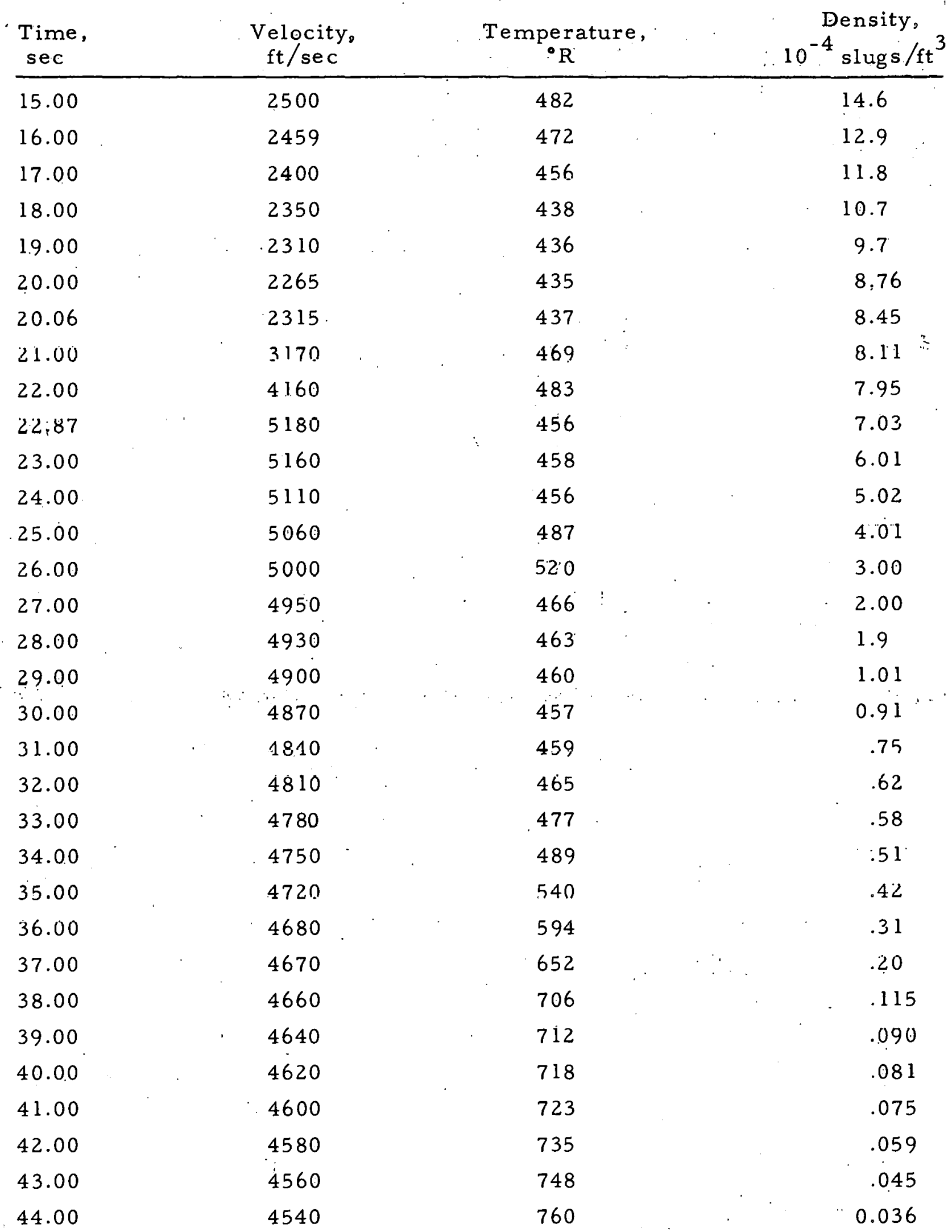




\begin{tabular}{cccc}
$\begin{array}{c}\text { Time } \\
\text { sec }\end{array}$ & $\begin{array}{c}\text { Velocity, } \\
\text { ft/sec }\end{array}$ & $\begin{array}{c}\text { Temperature, } \\
{ }^{\circ} \mathrm{R}\end{array}$ & $\begin{array}{c}\text { Density, } \\
10^{-4} \text { slugs } / \mathrm{ft}^{3}\end{array}$ \\
\hline 45.00 & 4520 & 763 & 0.028 \\
46.00 & 4500 & 764 & .024 \\
47.00 & 4480 & 765 & .020 \\
48.00 & 4460 & 765 & .0136 \\
49.00 & 4430 & 765 & .0121 \\
50.00 & 4400 & 765 & .0112 \\
51.00 & 4370 & 745 & .0093 \\
52.00 & 4340 & 725 & .0084 \\
53.00 & 4310 & 704 & .0075 \\
54.00 & 4280 & 684 & .0066 \\
55.00 & 4250 & 677 & .0057 \\
56.00 & 4220 & 669 & .0048 \\
57.00 & 4190 & 659 & .0040 \\
58.00 & 4160 & 649 & 0.0034 \\
\hline
\end{tabular}

\section{$\underline{\text { HAD Configuration Data Input }}$}

$$
\begin{aligned}
C_{\mathrm{pw}}= & 0.3 \mathrm{Btu} / \mathrm{lb} \mathrm{m}^{-0} \mathrm{~F} \\
\rho_{\mathrm{w}}= & 118.56 \mathrm{lb} \mathrm{m} / \mathrm{ft}^{3} \\
\delta_{\mathrm{w}}= & 0.0104 \mathrm{ft} \\
C_{\mathrm{p}}= & 0.24 \mathrm{Btu} / 1 \mathrm{~b}^{-{ }^{\circ} \mathrm{R}} \\
\mathrm{e}= & 0.7 \\
\sigma= & 0.173 \times 10^{-8} \mathrm{Btu} / \mathrm{ft}^{2}-\mathrm{h}-{ }^{\circ} \mathrm{R} \\
\mathrm{k}= & 1.4 \\
\mathrm{x}= & \text { Eight locations measured in feet from the forward end of } \\
& \text { the nose. Numerical values are adjusted to obtain the } \\
& \text { estimated correct boundary layer thickness. }
\end{aligned}
$$




\title{
APPENDIX C
}

\section{TYPICAL DATA OUTPUT}

Turbulent Approximation, $\mathrm{T}_{\mathrm{w}}$ in ${ }^{\circ} \mathrm{R}$

\author{
$30^{\circ}$ Cone, $x=2$ in.
}

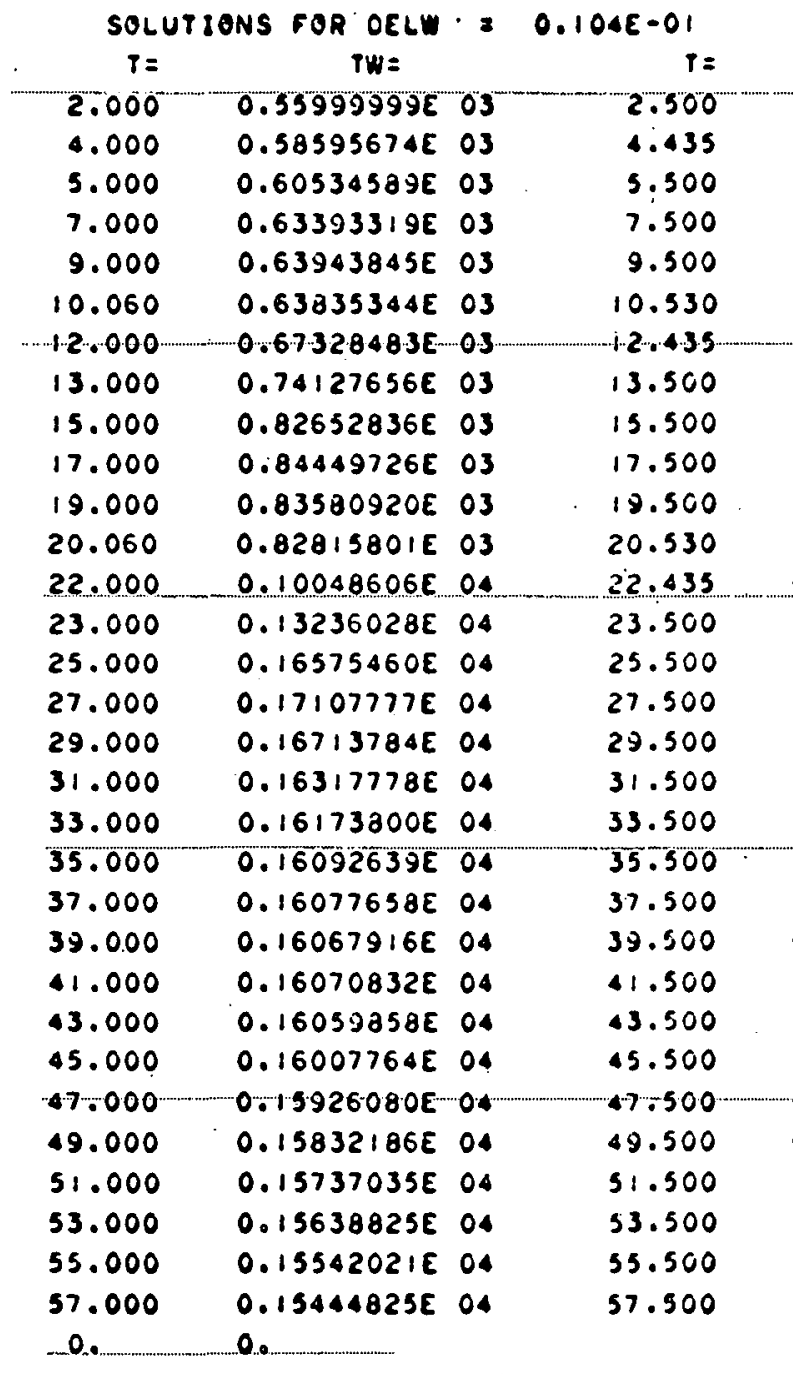

$x=0.057$

$$
T w=
$$
$T=$ $0.56293501 \mathrm{E} 03-3.000$ 0.59253532503 0.61593339503 $0.63677058 E$ O3 8.000 $0.63914035603 \quad: 0.000$ $0.63890968 E 03 \quad 11.000$ $0.69721394 E .03 \ldots 1.2 .870$ $0.83497749 E$ OS $\quad 16.000$ $0.84383338 E$ O3 $\quad 18.000$ $0.83210620 E$ O3 20.000 $0.83132713 E \quad 03 \quad 21.000$ $0.11235996 E$ 04 22.870 $0.14600500 E$ O4 24.000 $0.16871770 E$ OS 26.000 $0.17003057 E 04 \quad 28.000$ $0.16611773 E 04 \quad 30.000$ $0.16 .274571 E$ O4 32.000 $0.16147619 E \quad 04 \quad 35.000$ $0.16084183604 \quad 36.000$ $0.16077472 E$ O4 $\quad 33.000$ $0.16066917 E$ OS $\quad 40.000$ $0.16072876 E \quad 04 \quad 42.000$ $0.16050696 E$ O4 44.000 $0.15989297 E$ 04 46.000 TW $w=$ $T=\quad T H$ $0.56969132503 \quad 3.500-0.57867745 E 0.3$

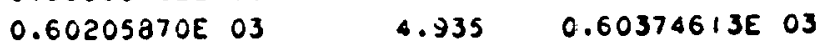
$0.62389412 E \quad 03 \quad 6.500 \quad 0.62975182 E \quad 03$ $0.63844430 E$ O3 $\quad 8.500 \quad 0.63925124 E 03$ $0.63845935 E$ OS $\quad 10.030 \quad 0.63840521 E 03$ $0.64367231 E 03 \quad 11.500 \quad 0.65475329 E^{\circ} 03$

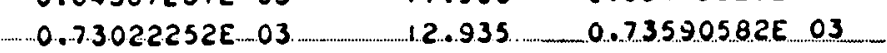

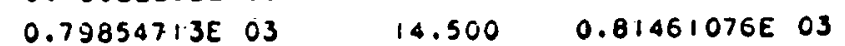
$0.84049521 E$ O3 $16.500 \quad 0.84357368 E \quad 03$ $\begin{array}{llllll}0.84196328 E & 03 & 18.500 & 0.83922672 E & 03\end{array}$

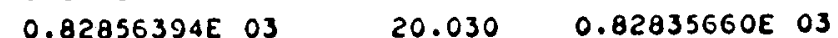

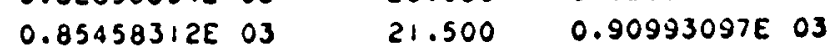
$0.12759852 E \quad 04 \quad 22.935 \quad 0.13010129 E 04$ $0.16146481 E 04$ $0.15527596 E 04 \quad 24.500$ $0.17068759 E 04 \quad 26.500$ $0.16897806 E \quad 04 \quad 28.500$ $0.16486485 E$ O4 28.500
30.500 0.1623676 .0 OS 32.500 $0.16124779 E 04 \quad 34.500$ $0.16146481 E$
$0.17154042 E$ $0.16799276 E$ O4 $0.16382383 E \quad 04$ $0.16203386 E 04$ $0.16106012 E \quad 04$ $0.16079520 E$ OA $\quad 36.500-0.16077512 E-04-$ $0.16074550 E$ O4 $0.16066906 E \quad 04$ 38.500 $0.16066906 E$ O4 40.500 $0.16070302 E 04$ $0.16068069 E 04$ $0.16066603 E$ O4 $0.16024361 E 04$ $0.15948337 E \quad 04$ $0.0 .58 .55899 E_{0.4}$ 42.500 $0.15963012 E .04 \ldots . .4000$ 0.15 .879 .568 .0 .04 $0.15712816 E$ O4 52.000 $0.15614630 E \quad 04 \quad 54.000$ $0.15784718 E$ O4 50.500 $0.15760940 E$ O4 52.500 54.500 $0.15517768 E$ O4 56.000 $0.15493426 E \quad 04$ 56.500 $0.15663242 E \quad 04$ $0.15566228 E 04$ $0.13420660 E 04 \quad 58.000$ $0.15396586 E$ OC

0 .

$0.15469081 E 04$ 0 . 
$20^{\circ}$ Cone, $x=4$ in.

\begin{tabular}{|c|c|c|c|c|c|c|c|c|}
\hline SOLU & $\begin{array}{c}\text { NS FOR DELWW } \\
\text { TW }=\end{array}$ & $\begin{array}{r}0.104 E-02 \\
T=\end{array}$ & $\begin{array}{c}x=0.111 \\
T H=\end{array}$ & $-T=$ & $T H=$ & $T=$ & $T W=$ & \\
\hline 2.000 & $0.55999999 E 03$ & 2.500 & $0.56249168 E 03$ & 3.000 & $0.568: 1973 E 03$ & 3.500 & $0.57515539 E$ & 03 \\
\hline 4.000 & $0.57995974 E 03$ & 4.435 & $0.58412082 E 03$ & 4.870 & $0.59142663 E 03$ & 4.935 & $0.59282918 E$ & 03 \\
\hline 5.000 & $0.59415494 E 03$ & 5.500 & $0.60305724 E \quad 03$ & 6.000 & $0.61001644 \mathrm{E} 03$ & 6.500 & $0.61542723 E$ & 03 \\
\hline 7.000 & $0.61961626 \mathrm{E} \mathrm{O3}$ & 7.500 & $0.62271996 E$ O3 & 8.000 & $0.62483500 E 03$ & 8.500 & $0.62614816 E$ & 03 \\
\hline 9.000 & $0.62683708 E 03$ & 9.500 & $0.62701661 E D 3$ & 10.000 & $0.62676746 E \quad 03$ & 10.030 & $0.62673992 E$ & 03 \\
\hline 20.060 & $0.62671690 . E 03$ & -10.530 & $-0.627 .46944 . E-03$ & -11.0000 & $0.631 .54269 \mathrm{E} 03$ & -11.500 & $0.64043 .244 E$ & 03 \\
\hline 12.000 & $0.6545918 E E_{03}$ & 12.435 & $0.67246819 E 03$ & 12.670 & $0.69619336 E 03$ & 12.935 & $0.70025131 \mathrm{E}$ & 03 \\
\hline 13.000 & $0.70414025 E 03$ & 13.500 & $0.73036169 E \quad 03$ & 14.000 & $0.75063041 E 03$ & 14.500 & $0.76637424 E$ & 03 \\
\hline 15.000 & $0.77872711 E 03$ & 15.500 & $0.78830859 E \quad 03$ & 16.000 & $0.79569982 E 03$ & 16.500 & $0.80117346 E$ & 03 \\
\hline 27.060 & $0.00483520 E 03$ & 17.500 & $0.80724153 E 03$ & 18.000 & $0.80864736 E 03$ & 18.500 & $0.80924662 E$ & 03 \\
\hline 19.000 & $0.80914795 E 03$ & 19.500 & $0.80858266 E 03$ & 20.000 & $0.80778889 E 03$ & 20.030 & $0.80774306 E$ & 03 \\
\hline 20.060 & $0.80772188 E 03$ & 20.530 & $0.81105214 E 03$ & 21.000 & $0.82515994 E \quad 03$ & 21.500 & $0.85638107 E$ & 03 \\
\hline 22.000 & $0.90933570 E 03$ & 22.43 .5 & $0.97682333 E 03$ & 22.870 & $0.10662325 E 04$ & 22.935 & $0.10811433 E$ & 04 \\
\hline 23.000 & $0.1094 \mathrm{R} 301 \leq 04$ & 23.500 & $0.11886919 E \quad 04$ & 24.000 & $0.12685440 E 04$ & 24.500 & $0.13348951 E$ & \\
\hline$\approx 5.000$ & $0.13889986 E$ U.4 & 25.500 & $0.14331175 E 04$ & 26.000 & $0.14689314 E 04$ & 26.500 & $0.14973944 E$ & \\
\hline 27.000 & $0.1519152 .6 E 04$ & 27.500 & $0.35358879 E \quad 04$ & 28.000 & $0.15490399 E 04$ & 28.500 & $0.15588956 E$ & \\
\hline 29.500 & $0.15657894 E 04$ & 29.500 & 0.15689360 E 04 & 30.000 & $0.15682100 E 04$ & 30.500 & $0.15663404 E$ & \\
\hline 31.000 & $0.15651189 E 04$ & 31.500 & $0.15640505 E \quad 04$ & 32.000 & $0.15626387 E 04$ & 32.500 & $0.15609675 E$ & \\
\hline 33.000 & $0.15591129 E 04$ & 33.500 & $0.15571290 E 04$ & 34.000 & $0.15550739 E 04$ & 34.500 & $0.15530 .179 E$ & 04 \\
\hline 35.000 & $0.15510315 E 04$ & 35.500 & $0.15490615 E \quad 04$ & 36.000 & $0.15470662 E 04$ & 36.500 & $0.25450592 E$ & \\
\hline 37.500 & $0.15430481 E 04$ & 37.500 & $0.15410156 E 04$ & 38.000 & $0.15389796 E 04$ & 38.500 & $0.15369707 E$ & \\
\hline 39.000 & $0.15350232 E 04$ & 39.500 & $0.15331368 E$ O4 & 40.000 & $0.15313489 E Q 4$ & 40.500 & $0.15296533 E$ & \\
\hline 41.000 & $0.15280427 E 04$ & 41.500 & $0.15264719 E$ O4 & 42.000 & $0.15248831 E 04$ & 42.500 & $0.15232731 E$ & \\
\hline 43.000 & $0.15216374 E 04$ & 43.500 & $0.15199771 E 04$ & 44.000 & $0.15183033 E 04$ & 44.500 & $0.15165996 E$ & 04 \\
\hline$-45 \div 000^{-}$ & $0.15146465 E 04$ & 45.500 & $0.15130498 E^{04}$ & 46.000 & $0.15112225 E 04$ & 46.500 & $0.15093663 E$ & 04 \\
\hline 47.000 & $0.15074818 E 04$ & 47.500 & $0.15055776 E 04$ & 48.500 & $0.15036688 E 04$ & 48.500 & $0.15017503 \mathrm{E}$ & \\
\hline 49.000 & $0.14998206 E 04$ & 49.500 & $0.14978804 E \quad 04$ & 50.000 & $0.14959313 E 04$ & 50.500 & $0.149397 \pi E$ & \\
\hline 51.000 & $0.14920215 E 04$ & 51.500 & $0.14900630 E 04$ & $52 . c 00$ & $0.14881001 E 04$ & 52.500 & $0.14661353 \mathrm{E}$ & \\
\hline 53.000 & $0.148417128 E 04$ & 53.500 & $0.14822078 E 04$ & $54 . c 00$ & $0.14802407 E 04$ & 54.500 & $0.14782737 E$ & \\
\hline 55.000 & $0.14763101 E 04$ & 35.500 & $0.14743495 E 04$ & 56.000 & $0.14723918 E$ O4 & 56.500 & $0.24704366 E$ & \\
\hline$-57-000$ & $0.2468848348-04$ & -57.500 & $-0.84665339 E^{04}$ & 68.000 & 0.846438660804 & ד & -0 & \\
\hline
\end{tabular}


$20^{\circ}$ Cone, $x=6$ in.

\begin{tabular}{|c|c|c|c|c|c|c|c|c|}
\hline \multicolumn{2}{|c|}{-SOLUTHONS-FOR-LELW- } & & & & \multirow{2}{*}{\multicolumn{3}{|c|}{$T h=$}} \\
\hline $\begin{array}{r}\text { SOEL } \\
T=\end{array}$ & $\begin{array}{c}\text { OKS-FOR-CELW- } \\
\text { TW }=\end{array}$ & $T=$ & $\begin{array}{l}O=166 \\
T W=\end{array}$ & $T=$ & $T W=$ & & & \\
\hline 2.000 & Q.55999999E 03 & 2.500 & $0.56227483 E$ G3 & 3.000 & $0.56740087 E 03$ & 3.500 & $0.57385860 E$ & 03 \\
\hline 4.060 & $0.57 .829693 E 03$ & 4.435 & $0.58216234 E \quad 03$ & 4.870 & $0.58900788 E 03$ & 4.935 & $0.59033024 E$ & 03 \\
\hline 5.000 & $0.59157954 E 03$ & 5.500 & $0.59998643 E 03$ & 6.000 & $0.60659605 E 03$ & 6.500 & $0.61177205 E$ & $03^{\circ}$ \\
\hline .7 .000 & $0: 61581705 E 03$ & 7.500 & $0.6: 885728 E .03$ & 8.000 & $0.62098587 E 03$ & 8.500 & $0.62237833 E$ & 03 \\
\hline.- .9 .000 & $0.623 .1 .91 .0 .7 . E-G 3$ & 9.500 & $-0.62 .35290 \div \cdot E-03-$ & $-10 \div 000$ & $0.62 \cdot 3.467 .76 E-0.3$ & -10.030 & $0.6234 .5228 \dot{E}$ & - \\
\hline 10.060 & $0.62344106 E 03$ & 10.530 & $0.62428662 E 03$ & 11.000 & $0.62817422 E 03$ & 11.500 & $0.65656859 E$ & 03 \\
\hline $12 \cdot 500$ & $0.65005705 E$ G3 & 12.435 & $0.66697086 \mathrm{E} .03$ & 12.870 & $0.68963271 \mathrm{E} 03$. & 12.935 & $0.69351826 E$ & 03 \\
\hline 12.000 & $0.69724243 E 03$ & 13.500 & $0.72240302 E 03$ & 14.000 & $0.74194308 E 03$ & 14.500 & $0.75721449 E$ & C3 \\
\hline 15.000 & $0.76929431 E 03$ & 15.500 & $0.77876741 E$ O3 & 16.500 & $0.78617372 E 03$ & 16.500 & $0.79176776 E$ & 03 \\
\hline 17.000 & $0.79570881 E 03$ & 17.500 & $0.79837724 E 03$ & 18.000 & $0.80013594 E 03$ & 18.500 & $0.80111704 E$ & 03 \\
\hline 29.000 & $0.80142231 E 0.3$ & 19.500 & $0.80 .126278 E 03$ & 20.000 & $0.8008580 .8 E 03$ & 20.030 & $0.80583495 E$ & 03 \\
\hline 20.060 & $0.80083593 E 03$ & 20.530 & $0.80423675 E 03$ & 21.000 & $0.81754714 E 03$ & 21.500 & $0.84665013 E$ & 03 \\
\hline $22 \cdot 000$ & $0.89592030 E 03$ & 22.435 & $0.55 E S 1294 E 03$ & $22.8 \pi$ & $0.10423972 E 04$ & 22.935 & $0.10563713 E$ & 04 \\
\hline 23.000 & $0.10692299 E 04$ & 23.500 & $0.1: 5 \& 2792 E$ D4 & $24 \cdot 000$ & $0.12352882 E 04$ & 24.500 & $0.13000684 E$ & 04 \\
\hline 25.000 & $0.13534077 E 04$ & 25.500 & $0.13973353 E 04$ & 26.000 & $0.14333696 E 04$ & 26.500 & $0.14623428 E$ & 04 \\
\hline 27.000 & $0.1 .4848422 E 04$ & 27.500 & $0.15024513 E 04$ & 28.000 & $0.15165295 E 04$ & 28.500 & $0.25273005 E$ & 04 \\
\hline 29.000 & $0.25350594 E 04$ & 29.500 & $0.15391033 E \quad 04$ & 30.000 & $0.15393236 E \quad 04$ & 30.500 & $0.15382852 \mathrm{E}$ & 04 \\
\hline 3.1 .000 & $0.15377026 E 04$ & 31.500 & $0.15371585 E 04$ & 32.000 & $0.153624 B O E$ O4 & 32.500 & $0.15350600 \mathrm{E}$ & 04 \\
\hline 33.000 & C. $15336776 E 04$ & 33.500 & $0.15321515 E \quad 04$ & 34.000 & $0.15305366 E 04$ & 34.500 & $0.15288918 E$ & 04 \\
\hline 35.000 & $0.15272737 E 04$ & 35.500 & $0.15256348 E$ O4 & 36.000 & $0.15239416 E 04$ & 36.500 & $0.15222073 E$ & E4 \\
\hline 37.000 & $0.15204324 E 04$ & 37.500 & $0.15186077 E 04$ & 38.000 & $0.15167645 E 04$ & 38.500 & $0.15149342 E$ & 04 \\
\hline 39.500 & $0.15131506 E 04$ & 39.500 & $0.15114170 E 04$ & 40.000 & $0.15097730 E 04$ & $40 \cdot 500$ & $0.15082140 E$ & 04 \\
\hline 41.000 & $0.15067343 E 04$ & 41.500 & $0.15052896 \mathrm{E} \quad 04$ & 42.000 & $0.15038237 E 04$ & 42.500 & $0.25023343 E$ & 04 \\
\hline 43.000 & $0.15008176 E 04$ & 43.500 & $0.14992 .749 \mathrm{E} 04$ & 44.000 & $0.14977164 E 04$ & $44.500^{-}$ & $0.14961272 E$ & 04 \\
\hline 45.060 & $0.14944893 E 04$ & 45.500 & $0.14928076 E 04$ & 46.000 & 0.1491094 EE 04 & 46.500 & $0.14893523 E$ & 64 \\
\hline 47.000 & $0.14875811 E 04$ & 47.500 & $0.14857894 E$ O4 & 48.000 & $0.14839917 E 04$ & 48.500 & $0.14821840 E$ & 04 \\
\hline 49.000 & $0.14803652 E 04$ & 49.500 & $0.14785358 E$ O4 & 50.000 & $0.14766975 E 04$ & 50.500 & $0.14748535 E$ & 04 \\
\hline 51.000 & 0.14730078804 & 51.500 & $0.14711586 E$ O4 & 52.000 & $0.14693043 E 04$ & 52.500 & $0.14674476 E$ & 04 \\
\hline 53.000 & $0.14655912 E 04$ & 53.500 & $0.14637335 E \quad 04$ & 54.000 & $0.14618725 E 04$ & 54.500 & $0.14600108 E$ & 04 \\
\hline 55.000 & $0.14581516 E^{0} 04$ & $55.500^{-1}$ & $0.14562945 \mathrm{E} 04$ & 56.000 & $0.14544395 E_{04}$ & 56.500 & $0.14525862 E$ & 04 \\
\hline 57.000 & $0.14507341 E 04$ & 57.500 & $0.14488841 E 04$ & 58.000 & $0.14470372 E 04$ & 0 & 0. & \\
\hline
\end{tabular}


$20^{\circ}$ Cone, $x=8$ in.

SOLUT IONS FOR DELW $=0.104 E-01$

$x=0.222$

\begin{tabular}{|c|c|c|c|c|c|c|c|c|}
\hline$T$ & $T W \equiv$ & $T \equiv$ & $T W=$ & $T \equiv$ & $T H=-$ & $T=$ & & \\
\hline 2.000 & $0.55999999 E 03$ & 2.500 & $0.56213262 E \quad 03$ & 3.000 & $0.56702675 E 03$ & 3.500 & $0.57321465 E$ & 03 \\
\hline 4.500 & $0.57747152 E 03$ & 4.435 & 0.58119698E 03 & 4.870 & $0.58778 .604 E 03$ & 4.935 & $0.50905789 E$ & 03 \\
\hline 5.000 & $0.59026065 E 03$ & 5.500 & $0.59837603 E 03$ & 6.000 & $0.60478774 E 03$ & 6.500 & $0.60983627 E$ & G3 \\
\hline 7.000 & $0.6138070 \mathrm{SE} 03$ & 7.500 & 0.61581750 E 03 & 8.000 & $0.61895177 E 03$ & 8.500 & $0.62037264 E$ & 03 \\
\hline $9 \cdot 000$ & $0.62123421 E 03$ & 9.500 & $0.62164064 E 03$ & 10.000 & $0.62166776 E 03$ & 10.030 & $0.62165 \mathrm{~S} 28 \mathrm{E}$ & 03 \\
\hline-10.060 & $-0.62165278 E 03$ & $10 \div 530$ & $0.62253441 \cdot E_{03}$ & $11 \cdot 000$ & $0.62633875 E_{03}$ & 11.500 & $0.634471-57 . E$ & -03 \\
\hline 12.000 & $0.64751022 E 03$ & 12.435 & $0.66382549 E \mathrm{CO}$ & $12 \cdot 3 \pi 0$ & $0.65570652 E 03$ & 12.935 & $0.68945999 E$ & 03 \\
\hline 13.000 & $0.69306065 E 03$ & 13.500 & $0.71746223 E 03$ & 14.000 & $0.73652953 E 03$ & 14.500 & $0.75152910 E$ & 03 \\
\hline $25 \cdot 000$ & $0.76342029 E 03$ & 15.500 & $0.77292756 E 03$ & 16.000 & $0.78037615 E 03$ & 16.500 & $0.76606927 E$ & 03 \\
\hline 17.000 & $0.79016151 E 03$ & 17.500 & $0.79300196 E 03$ & 18.000 & $0.79494303 E 03$ & 18.500 & $0.79611846 E$ & 03 \\
\hline$: 9.000$ & $0.79663280 E 03$ & 19.500 & $0.79669005 E 03$ & 20.000 & $0.79649952 E 03$ & 20.030 & $0.79648893 E$ & 03 \\
\hline 20.00 & $0.79650166 E_{03}$ & 20.530 & $0.75987948 E \quad 03$ & 21.000 & $0.81262212 E 03$ & -21.500 & $0.84041183 E$ & 03 \\
\hline 22.000 & $0.88747045 E 03$ & 22.435 & $0.94758112 E 03$ & 22.370 & $0.10274784 E 04$ & 22.935 & $0.1 \mathrm{C} 408303 \mathrm{E}$ & 04 \\
\hline 23.000 & $0.1053102 \mathrm{BE} 04$ & 23.500 & $0.11379705 E 04$ & 24.000 & $0.12122871 E 04$ & 24.500 & $0.12754297 E$ & 04 \\
\hline 25.000 & $0.1327 \approx 036 E 04$ & 25.500 & $0.13712559 E 04$ & 26.000 & $0.14071867 E 04$ & 26.500 & $0.14363410 E$ & 04 \\
\hline 27.000 & $0.14592078 E 04$ & 27.500 & $0.14773272 E 04$ & 28.000 & $0.14919792 E 04$ & 28.500 & $0.15033384 E$ & 04 \\
\hline 29.000 & G.15116661E 04 & $29 \cdot 500$ & $0.15163144 E 04$ & 30.000 & $0.15171844 E D 4$ & 30.500 & $0.15167214 \mathrm{E}$ & 04 \\
\hline 31.000 & $0.15165845 E$ O4 & 31.500 & $0.15164077 E 04$ & 32.000 & $0.15158495 E 04$ & 32.500 & $0.15150020 E$ & 04 \\
\hline 33.000 & $0.15139537 E 04$ & 33.500 & $0.15127527 E 04$ & 34.000 & $0.15114512 E 04$ & 34.500 & $0.15101002 E$ & 04 \\
\hline 35.000 & $0.15087469 E 04$ & 35.500 & $0.15073479 E 04$ & 36.000 & $0.15 C 58742 E 04$ & 36.500 & $0.15043389 E$ & 04 \\
\hline 37.000 & $0.15027421 E 04$ & 37.500 & $0.15010785 E 04$ & 38.000 & $0.14993847 E 04$ & 38.500 & $0.14976903 E$ & 04 \\
\hline 39.000 & $0.14960292 E 04$ & 39.500 & D.149440TOE 04 & 40.000 & $0.14928680 E 04$ & 40.500 & $0.24914093 E$ & 04 \\
\hline 41.000 & D.1490026AE OA & 41.500 & $0.14866755 E 04$ & 42.000 & $0.14873014 E 04$ & 42.500 & $0.14859026 \mathrm{E}$ & 04 \\
\hline 43.500 & $0.14844760 E 04$ & 43.500 & $0.14830219 E 04$ & 44.000 & $0.14815510 E 04$ & 44.500 & $0.14800490 E$ & 04 \\
\hline 45.000 & $0.1475498 B E 04$ & 45.500 & $0.14769046 E 04$ & 46.000 & $0.14752792 E 04$ & 46.500 & $0.14736236 \mathrm{E}$ & 04 \\
\hline ט. 47.00 & $0.14719389 E 04$ & 47.500 & $0.14702331 E 04$ & 48.000 & $0.14685204 E 04$ & 48.500 & $0.14667971 E$ & 04 \\
\hline 49.000 & $0.14650627 E 04$ & 49.500 & $0.14633178 E 04$ & 50.000 & $0.14615636 E 04$ & 50.500 & $0.14598036 E$ & 04 \\
\hline 51.000 & $0.14580415 E 04$ & $51.500^{\circ}$ & $0.14562756 E D 4$ & 52.060 & $0.14545043 E 04$ & 52.500 & $0.14527299 E$ & 04 \\
\hline 53.000 & $0.14509553 E 04$ & 53.500 & $0.14491791 E 04$ & 54.000 & $0.14473989 E 04$ & 54.500 & $0.14456276 E$ & 04 \\
\hline 55.000 & $0.14438381 E 04$ & 55.500 & $0.14420602 E 04$ & 56.000 & $0.14402837 E 04$ & 56.500 & $0.24385082 E$ & 04 \\
\hline $57: 000$ & Q. $14367333 E^{\circ} 04$ & 57.509 & $0.14349598 \mathrm{E} 04$ & $58.000^{-}$ & $0.14331889 E-04$ & 0 & $-0 \div$ & \\
\hline
\end{tabular}

0 .

D. 
$10^{\circ}$ Cone, $x=4$ in.

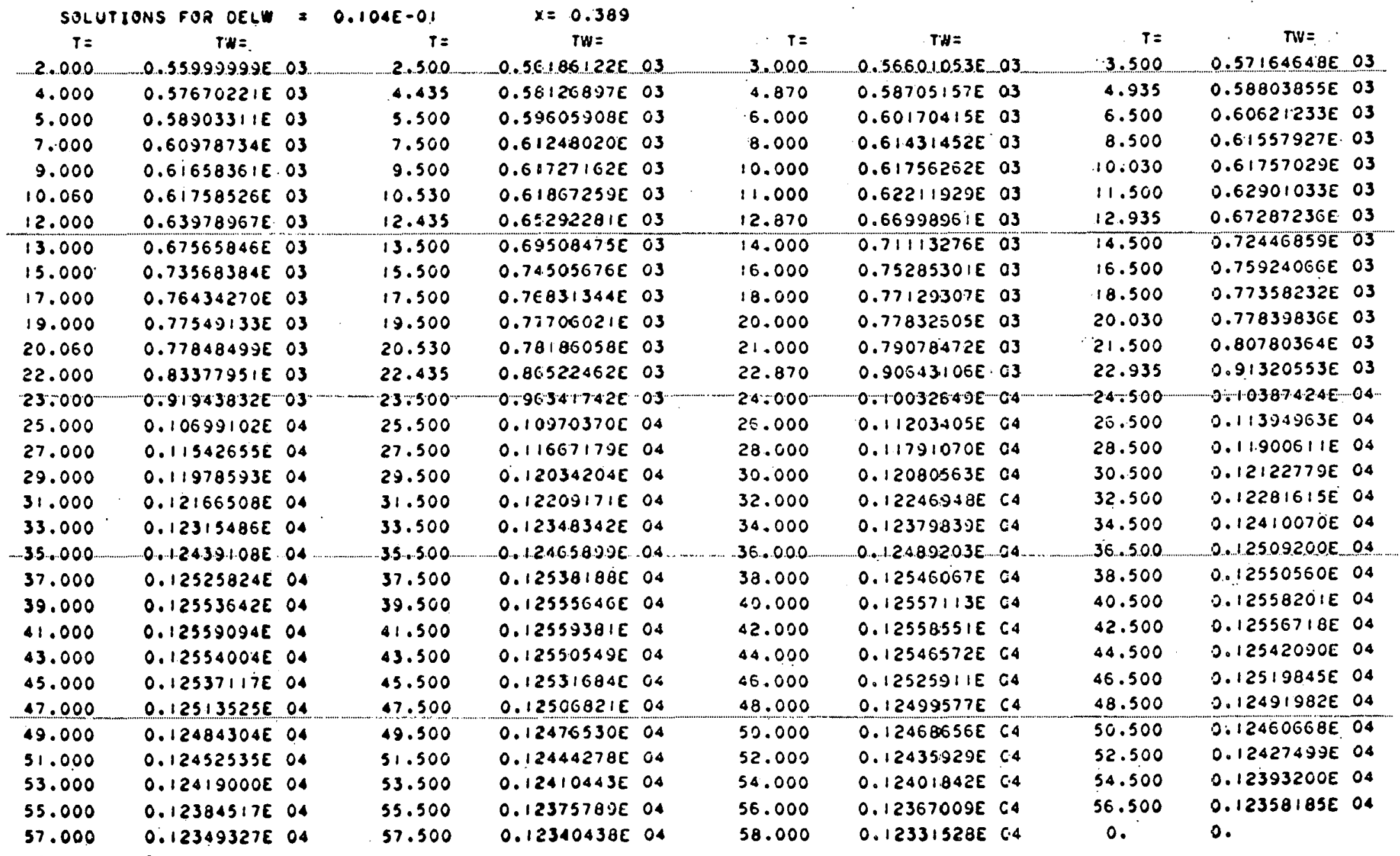


$0^{\circ}$ Cone, $x=18$ in.

\begin{tabular}{|c|c|c|c|c|c|c|c|c|c|c|c|}
\hline $\begin{array}{l}\text { SOLU } \\
?=\end{array}$ & $\begin{array}{c}\text { IS FOR CELH } \\
T W=\end{array}$ & $=$ & $\begin{array}{r}0.104 E-01 \\
T=\end{array}$ & $\begin{array}{c}x=0.301 \\
T W=\end{array}$ & & $T=$ & $T W=$ & & $i=$ & $T H=$ & \\
\hline 2.000 & & 03 & 2.506 & $0.5601043 \pi$ & 03 & 3.000 & $0.56099: 46 E$ & 03 & 3.3 .00 & $0.5630765 x$ & 23 \\
\hline $4.0,00$ & 0.566810158 & 03 & $4.43 E$ & $0.5 .7160635 E$ & 03 & 4.870 & $0.37732912 E$ & 03 & 4.935 & 0.578875355 & 03 \\
\hline 5.000 & $0.5798706 \alpha E$ & 03 & 5.300 & 0.586741948 & 3 & 6.000 & 0.592425756 & 03. & 6.500 & 0.597109555 & 03 \\
\hline 7.000 & $0.60006 / 810$ & 03 & $7.50 C$ & $0.6040451 \pi$ & 03 & B. 000 & $0.60541906 E$ & 03 & 0.300. & 0.60821102 & C3 \\
\hline 9.000 & $0.600563 A R E$ & 03 & 9.500 & 0.61 .0519185 & 03 & 10.000 & $0.6111200 x$ & 03 & 10.030 & 0.611147218 & 03 \\
\hline 10.060 & $0.61118045 \mathrm{~F}$ & 03 & 10.536 & $0.6124 B E A X$ & 03 & 11.000 & $0.6150320 x$ & .3 & $: i .500$. & $0.62248533 x$ & 03 \\
\hline 12.000 & 0.03244285 & 0.3 & $12 . .43 .5$ & $0.6442568 \pi$ & 0.2 & 12.870 & 0.65 .022557 & -0.3 & 12.935 & -0.66 .17 .4060 & 0.3 \\
\hline 13.000 & $0.6641181 x$ & 03 & 13.300 & 0.681077528 & 03 & 14.000 & $0.69541351 \pi$ & 03 & 14.500 & $0.707781 B E$ & $c 3$ \\
\hline 15.000 & $0.7183 B 060 x$ & 03 & 15.500 & a. $7274742^{\circ} \varepsilon$ & 03 & is. 000 & $0.735243 \mathrm{HE}$ & 03 & 16.300 & $0.7418 \angle 04 C E$ & 03 \\
\hline 17.000 & 0.747397425 & 03 & 17.300 & a. 752053002 & 0.3 & 18.000 & 0.755977 ISE & 03 & .18 .500 & 0.759228155 & 03 \\
\hline 19.000 & $0.76,9039 x$ & 0.3 & 19.500 & $0.761 / 493 x$ & 03 & 25.000 & $0.7 \in 6,121.9 E$ & 03 & 20.030 & 0.766225755 & 03 \\
\hline 20.060 & 0.766351358 & 03 & 25.335 & C. $7700565 x$ & 03 & 2.000 & $0.7783415 x$ & 03 & 21.500 & 0.793172468 & 03 \\
\hline 22.000 & 0.81494857 & 03 & 22.435 & C. BAOS6GBAC & 03 & 22.870 & $0.8715307 x$ & 03 & 22.935 & $0 . B T>7102 C E$ & 03 \\
\hline 23.000 & $0.8 B|B| 3 B E E$ & 03 & 23.300 & $0.91 B B A 795$ & 03 & 24.050 & 0.050902145 & 03 & 24.500 & 0.978272135 & 03 \\
\hline 25.000 & 0.100186282 & 04 & 25.500 & Q. $1021567 \mathrm{IE}$ & 04. & 25.000 & 0.103709535 & 04 & 25.300 & 0. 1C493594E & 04 \\
\hline 27.000 & 0. 10591729 & 04 & 27.500. & a. $1060300 x$ & 34 & 28.000 & 0.10770524 & 04 & 28.500 & 0.160566055 & 04 \\
\hline 29.000 & 0. $1093077 x$ & 04 & 29.500 & a. $1101213 / 5$ & 04 & 30.000 & 0.1 .10647312 & 04 & 30.500 & $0.11106373 x$ & 04 \\
\hline 31.000 & $0.1114761 x$ & 04 & 31.300 & $a+11 B G \| 15$ & 04 & 32.000 & $0.1121904 x$ & 04 & 32.500 & $0.11249656 E$ & 04 \\
\hline 33. 000 & $0.11275 \mathrm{BISE}$ & 04 & 33.300 & a. 1 । $29 B 99 x$ & 6: & 34. 300 & 0.1 .13200355 & 04 & 34.500 & O. $11339004 C E$ & 04 \\
\hline-35.000 & $0.1+35.628 x$ & -04 & $-35,-500$ & O. 1+1-37+5ose & -54 & $-35,-900$ & $0 . \div+33220 x$ & -04 & $36 .-500$ & $-0.17390838 \mathrm{C}$ & \\
\hline 37.020 & $0.1139816 x$ & 04 & 37.500 & a. $1140433 x$ & 04 & 38.000 & 0.114009635 & 04 & 36.900 & 0.114146565 & 04 \\
\hline 39.000 & $0.11417875 x$ & 04 & 39.500 & a 1141 gBaSE & 04 & 45.000 & $0.1142113 x$ & 04 & 50.300 & 0.114218556 & 04 \\
\hline 41.000 & $0.1142233 \mathrm{CE}$ & 04 & 41.500 & a. 114224635 & 04 & 42.000 & $0.1142218 \pi$ & 04 & 42.900 & 0.11421525 & 04 \\
\hline 43.000 & $0.114204 B C E$ & 04 & 43.500 & a. $1141901 x$ & 04 & 44.0000 & $0.114171,15$ & 04 & 44.900 & 0.114147555 & 04 \\
\hline 45.000 & $0.1141193 x$ & 04 & 45.500 & Q. $1140059 \pi$ & 01 & $45: 000$ & $0.1140467 x$ & 04 & 45.500 & 0.114004055 & 04 \\
\hline 47.000 & $0.113060 B C E$ & 04 & 47.500 & a 11301693 & 28 & 48.200 & $0.1138724 x$ & 04 & 48.500 & 0. 11382747 & 04 \\
\hline 49.000 & $0.11378235 E$ & 04 & 99.500 & $0.1137368 F E$ & 04 & 50.000 & 0.11369123 & 24 & 50.500 & $0.11364603 E$ & 04 \\
\hline 51.000 & 0.1130025 .18 & 04 & 51.500 & a $1135583 x$ & 04 & 52. 000 & 0.113510192 & 04 & 52.500 & 0.113459796 & 04 \\
\hline 53.050 & O. $1131085 A E$ & 04 & 53.900 & a.1133565BE & 04 & 54.000 & 0.11330412 & 04 & 54.500 & $0.11325111 E$ & 04 \\
\hline 55.000 & $0.1131977 x$ & 01 & 59.300 & a. $11314385 E$ & 04 & 56.000 & $0.1130092 x$ & 04 & 55.500 & $0.11303391 E$ & 04 \\
\hline 57.000 & $0.112977 B 5 E$ & 04 & 37.300 & a. $1129209 x$ & 08 & 58.000 & 0. 11286296E & 04 & j. & 0. & \\
\hline
\end{tabular}


$0^{\circ}$ Cone, $x=22$ in.

\begin{tabular}{|c|c|c|c|c|c|c|c|c|c|c|c|}
\hline $\begin{array}{l}\text { Sol. } \\
T=\end{array}$ & $\begin{array}{c}\text { NS FCR CELW } \\
\text { TW }=\end{array}$ & $=$ & $\begin{array}{r}0.104 E-0.1 \\
T=\end{array}$ & $\begin{array}{c}x=0.612 \\
T W=\end{array}$ & & $T=$ & $T W=$ & & $T=$ & $T W=$ & \\
\hline 2.000 & $0.35090000 x$ & 03 & 2.530 & $-0.360+1008 x$ & 0.3 & $3: 000$ & $-0.36009578 x$ & -0.3 & $-3 .-500$ & $0.5629720+15$ & -03 \\
\hline 4.000 & $0.9665 B 0346$ & 03 & 4.435 & $0.5712289 \pi$ & 03 & 4.370 & $0.3772748 x$ & 03 & 4. 935 & 0.578292248 & .03 \\
\hline 5.000 & $0.3792504 x$ & 03 & 3.350 & $0.5 B \leq 0 A 4 B X$ & 03 & 6.050 & $0.5914813 C$ & 03 & 6.500 & $0.59004991 E$ & 03 \\
\hline 7.000 & $0.599 B 1415 E$ & 03 & 7.500 & $0.60283 \div 545$ & 03 & B. B. B. & $0.605,60,35$ & 03 & 3.500 & $0.6069437 x$ & 03 \\
\hline 9.000 & 0. 608292?TE & 03 & 9.500 & 0.6052578 .56 & 03 & 10.000 & $0.609 B^{7} B S$ IE & 03 & 10.030 & 0.609307505 & 03 \\
\hline 10.060 & $0.60004106 E$ & 03 & 10.530 & 0.611243855 & 03 & סנספיוי & $0.6146055 x$ & 03 & 11.500 & $0.62 \cos 912 x$ & .03 \\
\hline 12.000 & $0.6307026 x E$ & 03 & 12.435 & $0.6422134 C E$ & 23 & 12. B 75 & $0.6567925 x$ & 03: & 12.935 & $0.65921650 c$ & 03 \\
\hline 13.000 & $0.66155856 E$ & 03 & 13.500 & $0.6780052 x$ & 23 & 1.4 .000 & 0.6921 G9SGE & 03 & 14.500 & 0.704202665 & 03 \\
\hline 15.000 & $0.7145 B 14 T E$ & 03 & 15.500 & $0.7234943 / 2$ & 03 & 15.000 & $0.73111936 C$ & 03 & 16.500 & 0.737602356 & 03 \\
\hline 17.000 & 0.743077556 & 03 & 17.500 & 0.74763965 & 03 & 18.500 & 0.731573348 & 03 & $=8.500$ & $0.75481246 E$ & 03 \\
\hline 19.000 & $0.757490,46 E$ & 03 & 10.500 & a. $75974803 E$ & 03 & 20.000 & 0.761729618 & 23 & 20.030 & $0.7618443 x$ & 03 \\
\hline 20.050 & $0.761 .97061 E$ & 03 & 20.930 & a. $7656377 C E$ & 03 & 21.090 & $0.773706: \pi$ & 03 & 21.500 & $0.78810173 E$ & 03 \\
\hline 22.000 & 0.809178250 & 03 & 22.135 & $0.8334584 x$ & 03 & 22.870 & $0.863 B A B A T E$ & 03. & 22.935 & $0.80585028 E$ & 03 \\
\hline$-23.950-$ & 0.873779565 & 03 & $-23.500-$ & $0.0095930 x$ & 0.5 & 24.000 & $0.9406224 x$ & 03 & $2 \% 500$ & $0.967 .96 \% 7 R$ & 03 \\
\hline 25.000 & $0.9900434 \pi$ & 03 & 25.900 & $0.10 .29 \pi 766 \varepsilon$ & 08 & 25.000 & $0.102426^{7} x$ & 04 & 26.500 & $0.10361964 \varepsilon$ & 04 \\
\hline 27.000 & $0.10460427 E$ & 04 & 27.500 & a. 105864325 & 04 & 28.000 & $0.1063176 \pi$ & .04 & 283.500 & $0.1071504 x$ & 04 \\
\hline 29.000 & $0.10794926 t$ & 04 & 29.500 & $0.1036439 x$ & 04 & 30.000 & $0.1091513 x$ & 04 & 30.500 & $0.10955773 E$ & 04 \\
\hline 31.000 & 0. $1090564 x$ & 04 & 31.500 & 0.11033092 & 08 & 32.000 & $0.1106605 x$ & 0.4 & 32.500 & $0.11 \cos 507 C E$ & 04 \\
\hline 33.000 & $0.11120676 E$ & 04 & 33.500 & $0.11145426 E$ & 04 & 34.000 & $0.1116411 x$ & 04 & 34.500 & 0.111828486 & 04 \\
\hline 35.000 & $0.111 .998 B 2 C$ & 04 & 35.500 & $0.112 .1647 x$ & 04 & 35.000 & $0.1-122563 x$ & 64 & -35.500 & 0.11 .2342 .955 & - 04 \\
\hline 37.000 & 0.112416905 & 0.4 & 37.500 & $0.11249958 E$ & 04 & 38.000 & $0.112536 .95 E$ & 04 & 3B. 500 & $0.1125 B 529$ & 04 \\
\hline 39.000 & 0.11261919 & 04 & 39.500 & $0.11264135 E$ & 04 & 40.000 & $0.112655, E E$ & 04 & 40.500 & $0.112865944 E$ & 04 \\
\hline 41.000 & $0.1126729 x$ & 0.4 & 41.500 & $0.1126767 x$ & 04 & 42.000 & $0.1126755 x$ & 04 & 42.500 & $0.11267281 E$ & 04 \\
\hline 43.000 & $0.11266321 E$ & 04 & 43.500 & a. $11265353 E$ & 04 & $: 44.000$ & $0.1 .126375 C E$ & 04 & 44.300 & $0.11261702 x$ & 04 \\
\hline 45.000 & 0.112591942 & 04 & 45.500 & Q. 112551618 & 04 & 46.000 & $0.112525 .54 C$ & 04 & 46.500 & $0.11248602 E$ & 04 \\
\hline 47.000 & 0.112446008 & OA & 47.500 & 0.112405415 & 04 & 48.000 & $0.11236120 x$ & 04 & 48.500 & 0.312322506 & 04 \\
\hline 49.000 & 0.112280755 & 04 & 49.500 & $0.1122386 \mathrm{RA}$ & 04 & 50.000 & $0.11219536 E$ & 04 & 50.300 & $0.111215451 E$ & 04 \\
\hline 51.000 & 0.112114356 & 04 & 51.500 & a. $11207335 E$ & 04 & 32.000 & $0.1120286 \pi$ & 04 & 52.500 & 0.111981758 & 04 \\
\hline 53.000 & 0.111933965 & 04 & 53.500 & $0.1118 B 553 E$ & 04 & 34.000. & $0.11183 E 5 x$ & 04 & 54.500 & $0.1117871 \pi$ & 04 \\
\hline 55.0 .00 & $0.1117373 x$ & 04 & 55.500 & 0.111636928 & 04 & 36.000 & $0.1116358 \mathrm{C}$ & 04 & 36.500 & $0.1115840 \pi$ & 04 \\
\hline 37.000 & $0.1115319 \pi$ & 04 & 37.300 & 0.11147822 & .04 & 58.000 & $0.1114230 x$ & 04 & 0. & 0. & \\
\hline
\end{tabular}


$30^{\circ}$ Cone, $x=2 \vdots n$.

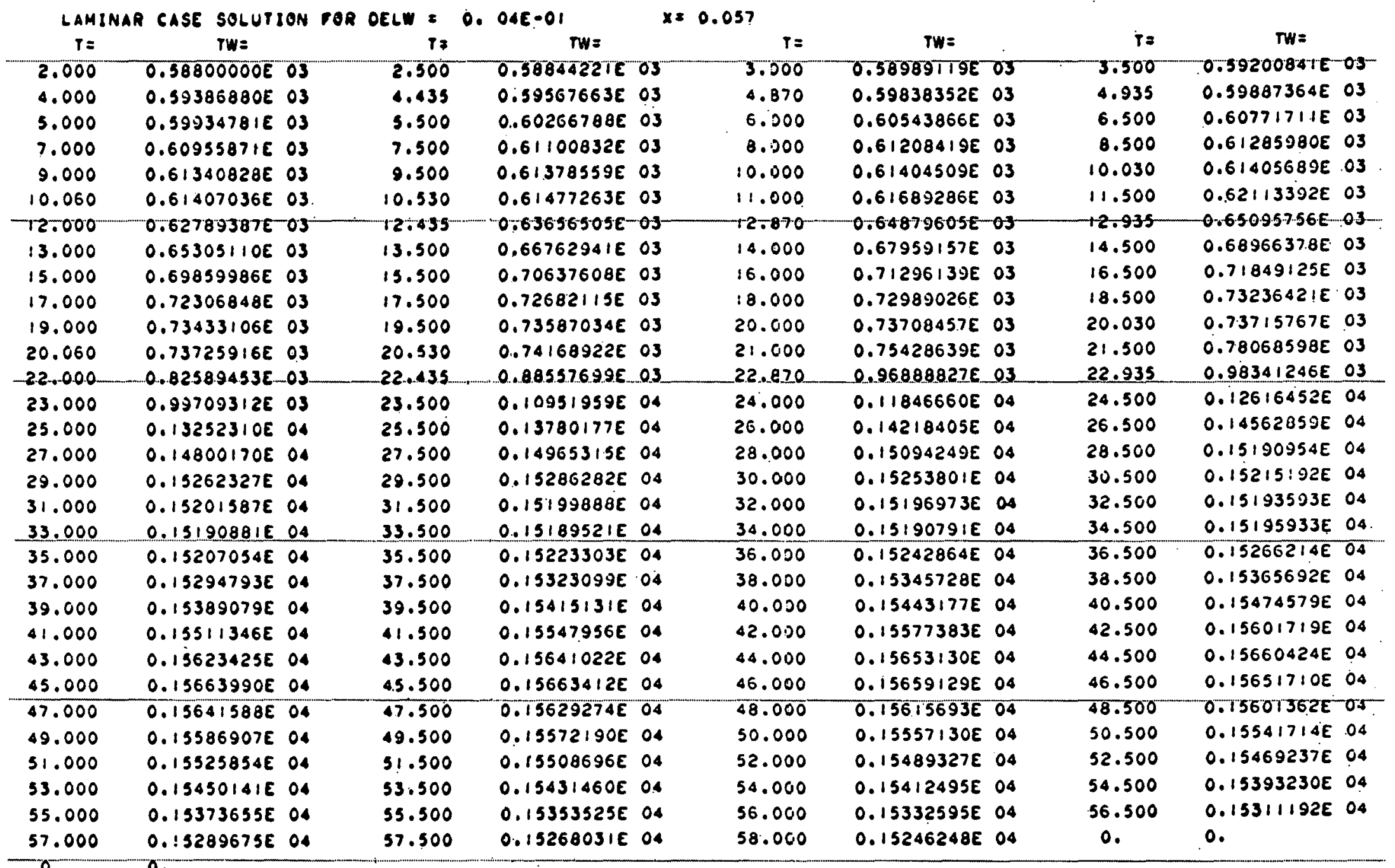


$20^{\circ}$ Cone, $x=4$ in.

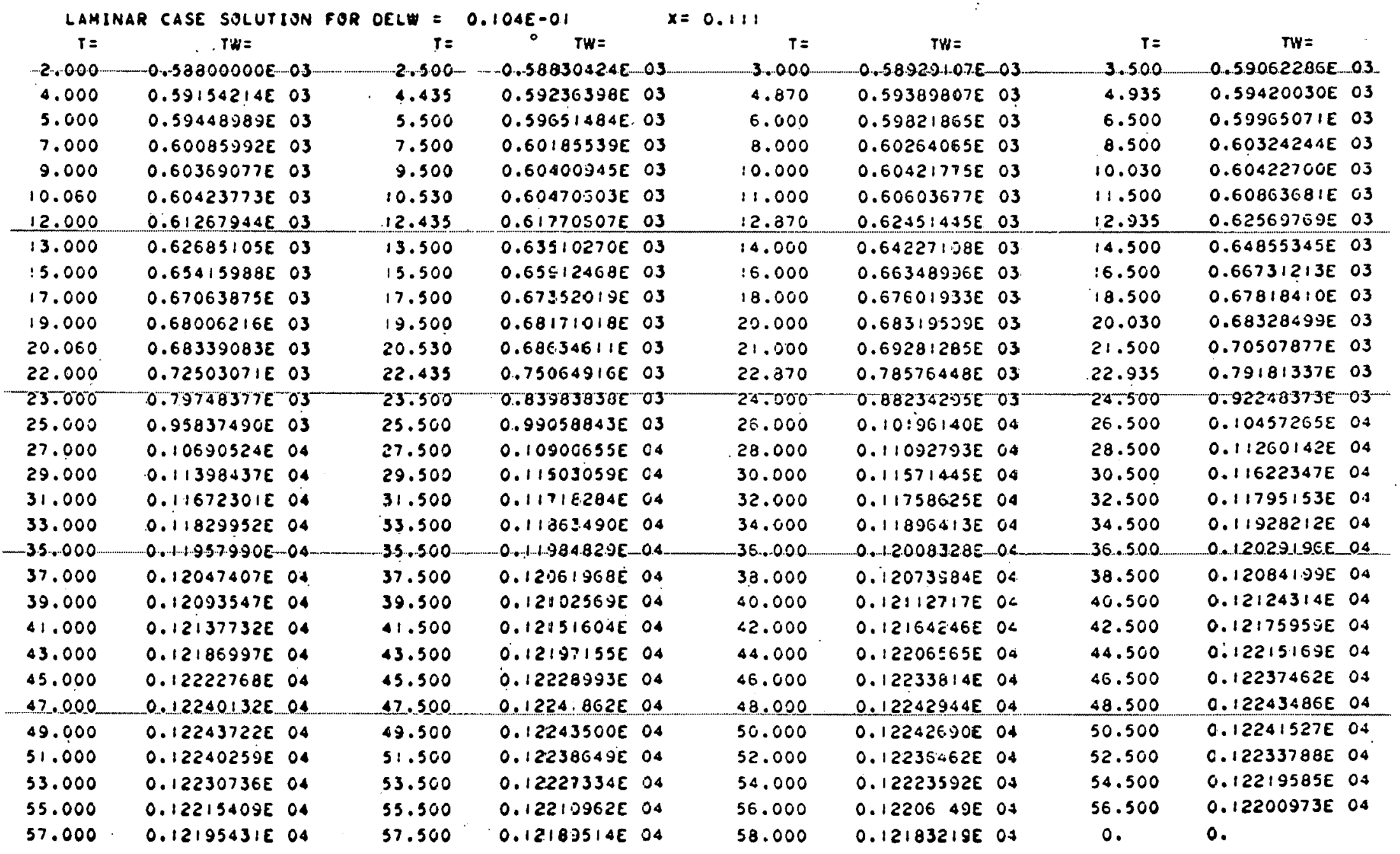

0 . 
$20^{\circ}$ Cone, $x=6$ in.

\begin{tabular}{|c|c|c|c|c|c|c|c|c|c|c|c|c|}
\hline $\begin{array}{l}\text { LAMINAR } \\
T=\end{array}$ & 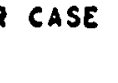 & $T W=$ & ION FOR & $\begin{aligned} \text { DELW } & = \\
r & =\end{aligned}$ & $\begin{array}{r}0.194 E-01 \\
T W=\end{array}$ & $x=$ & 0.166 & $T W=$ & & $T=$ & $r w=$ & \\
\hline 2.000 & 0.5880 & $00000 \varepsilon$ & 03 & 2.500 & $0.58824753 E$ & 03 & 3.000 & $0.58905463 \mathrm{E}$ & -03 & 3.500 & $0.590+4668 E$ & -0.3 \\
\hline 0.000 & 0.5909 & $90417 E$ & 03 & 4.435 & $0.59158347 E$ & 03 & 4.870 & $0.59284893 E$ & 03 & 4.935 & $0.59309818 \varepsilon$ & 03 \\
\hline 5.000 & 0.5933 & 337256 & 03 & 5.500 & $0.59501358 E$ & 03 & 6.000 & $0.59643100 E$ & 03 & 6.500 & $0.59762888 E$ & 03 \\
\hline 7.000 & 0.5986 & $64669 E$ & 03 & 7.500 & $0.59949125 E$ & 03 & 8.000 & $0.600: 6496 E$ & 03 & 8.500 & $0.60068910 E$ & 03 \\
\hline 9.000 & 0.6010 & $08763 E$ & 03 & 9.500 & $0.60138027 E$ & 03 & 10.000 & $0.60158333 E$ & 03 & 10.030 & $0.60159289 E$ & 03 \\
\hline 10.060 & 0.6016 & $60369 E$ & 03 & 10.530 & $0.60202060 E$ & 03 & $1: .000$ & $0.60314835 E$ & 03 & 11.500 & $0.60532583 E$ & 03 \\
\hline-12.000 & 0.6086 & $69728 E$ & -0.3 & 12.435 & $0.6 \cdot 1-2882.96 E$ & -0.3 & $-1-2-870$ & $0.61 .855+74 E$ & -03 & 12.035 & $-0.619 .5 .37 .2 .4 E$ & 03 \\
\hline 13.000 & 0.6204 & $49864 E$ & 03 & 13.500 & $0.6273991 .9 \varepsilon$ & 03 & 14.000 & $0.63343095 E$ & 03 & 14.500 & $0.63875115 E$ & 03 \\
\hline 15.000 & 0.6435 & $53091 E$ & 03 & 15.500 & $0.64779300 E$ & 03 & 16.000 & $0.65: 156608 E$ & 03 & 16.500 & $0.65489596 E$ & 03 \\
\hline 17.000 & 0.6578 & $82300 E$ & 03 & 17.500 & $0.66038489 \varepsilon$ & 03 & $: 8.000$ & $0.66262928 E$ & 03 & 19.500 & $0.66459545 E$ & 03 \\
\hline 19.000 & 0.6663 & $32330 E$ & 03 & 19.500 & $0.66785829 E$ & 03 & 20.000 & $0.66925452 E$ & 03 & 20.030 & $0.66933912 \varepsilon$ & 03 \\
\hline 20.060 & 0.6694 & $43769 E$ & 03 & 20.530 & $0.67206918 E$ & 03 & $2: .000$ & $0.67762308 E$ & 03 & $\hat{\imath .500}$ & $0.68801893 E$ & 03 \\
\hline-2.2 .0 .00 & 0.7048 & $8.347 .1 E$ & 0.3 & 22.435 & $0.72638497 E$ & 03 & 22.370 & $0.75592412 E$ & 03 & 22.935 & 0.761015398 & 03 \\
\hline 23.000 & 0.7657 & $79335 E$ & 03 & 23.500 & $0.80163400 E$ & 03 & 24.000 & $0.83789415 E$ & 03 & 24.500 & $0.87242676 E$ & 03 \\
\hline 25.000 & 0.9035 & $56077 E$ & 03 & 25.500 & 0.931732516 & 03 & 26.500 & $0.95732361 E$ & 03 & 26.500 & $0.98054846 E$ & 03 \\
\hline 27.000 & 0.1001 & $15044 E$ & 04 & 27.500 & $0,10205655 E$ & 04 & 28.500 & $0.10381446 E$ & 04 & 28.500 & $0.10535961 E$ & 04 \\
\hline 29.000 & 0.1066 & $54911 E$ & 04 & 29.500 & $0.10764793 E$ & 04 & 30.000 & $0.10833565 E$ & 04 & 30.500 & $0.10886307 E$ & .04 \\
\hline 31.000 & 0.1093 & $36768 E$ & 04 & 31.500 & $0.10982758 E$ & 04 & 32.000 & $0.11023653 E$ & 04 & 32.500 & $0.11061162 E$ & 04 \\
\hline 33.000 & 0.1109 & $97269 E$ & 04 & 33.500 & $0.11132360 E$ & 04 & 34.000 & $0.11166974 E$ & 04 & 34.500 & $0.11200488 E$ & 04 \\
\hline 35.000 & 0.1123 & $31854 E$ & 04 & 35.500 & $0.11260238 E$ & 04 & 36.000 & $0.11285314 E$ & 04 & 36.500 & $0.11307737 \mathrm{E}^{-}$ & -04 \\
\hline 37.000 & 0.1132 & $27423 E$ & 04 & 37.500 & $0.11343464 E$ & 04 & 38.000 & $0.11356964 E$ & 04 & 38.500 & $0.11368590 E$ & 04 \\
\hline 39.000 & 0.1137 & $79185 E$ & 04 & 39.500 & $0.11389308 E$ & 04 & 40.000 & $0.11400343 E$ & .04 & 40.500 & $0.11412602 E$ & 04 \\
\hline $4: .000$ & 0.1142 & $26439 E$ & 04 & 41.500 & $0.11440615 E$ & 04 & 42.000 & $0.11453617 E$ & 04 & 42.500 & $0.11465728 \mathrm{E}$ & 04 \\
\hline .43 .000 & 0.1147 & $77100 E$ & 04 & 43.500 & $0.11487804 E$ & 04 & 44.000 & $0.11497688 E$ & 04 & 44.500 & $0.11506815 E$ & 04 \\
\hline 45.000 & 0.1151 & $15037 E$ & 04 & 45.500 & $0.11522011 E$ & 04 & 46.000 & $0.11527701 E$ & 04 & $46.500^{\circ}$ & $0.11532313 E$ & 04. \\
\hline 87.000 & 0.1153 & $36024 E$ & 04 & 47.500 & $0.17538860 \mathrm{E}$ & 04 & 48.000 & $-0.175+1082 E$ & 04 & -48.500 & $-0.1+54-28+0 E$ & -04 \\
\hline 49.000 & 0.1154 & $44282 E$ & 04 & 49.500 & $0.11545358 E$ & 04 & 50.000 & $0.11545920 E$ & 04 & 50.500 & $0.11546174 E$ & .04 \\
\hline 51.000 & $0.1 ! 54$ & $46344 E$ & 04 & 51.500 & $0.11546217 E$ & 04 & 52.000 & $0.11545573 E$ & 04 & 52.500 & $0.11544493 E$ & 04 \\
\hline 53.000 & 0.1154 & $43065 E$ & 04 & 53.500 & $0.11541321 E$ & 04 & 54.000 & $0.11539283 E$ & 04 & 54.500 & $0.11537009 E$ & 04 \\
\hline 55.000 & 0.11 .53 & $34585 E$ & 04 & 55.500 & $0.11331910 E$ & 04 & 56.000 & $0.11528895 E$ & 04 & 56.500 & $0.11525545 E$ & 04 \\
\hline 57.000 & 0.1152 & $21861 \varepsilon$ & 04 & 57.300 & $0.11517826 E$ & 04 & 58.000 & $0.11513429 E$ & 04. & 0. & 0. & \\
\hline
\end{tabular}


$20^{\circ}$ Cone, $x=8$ in.

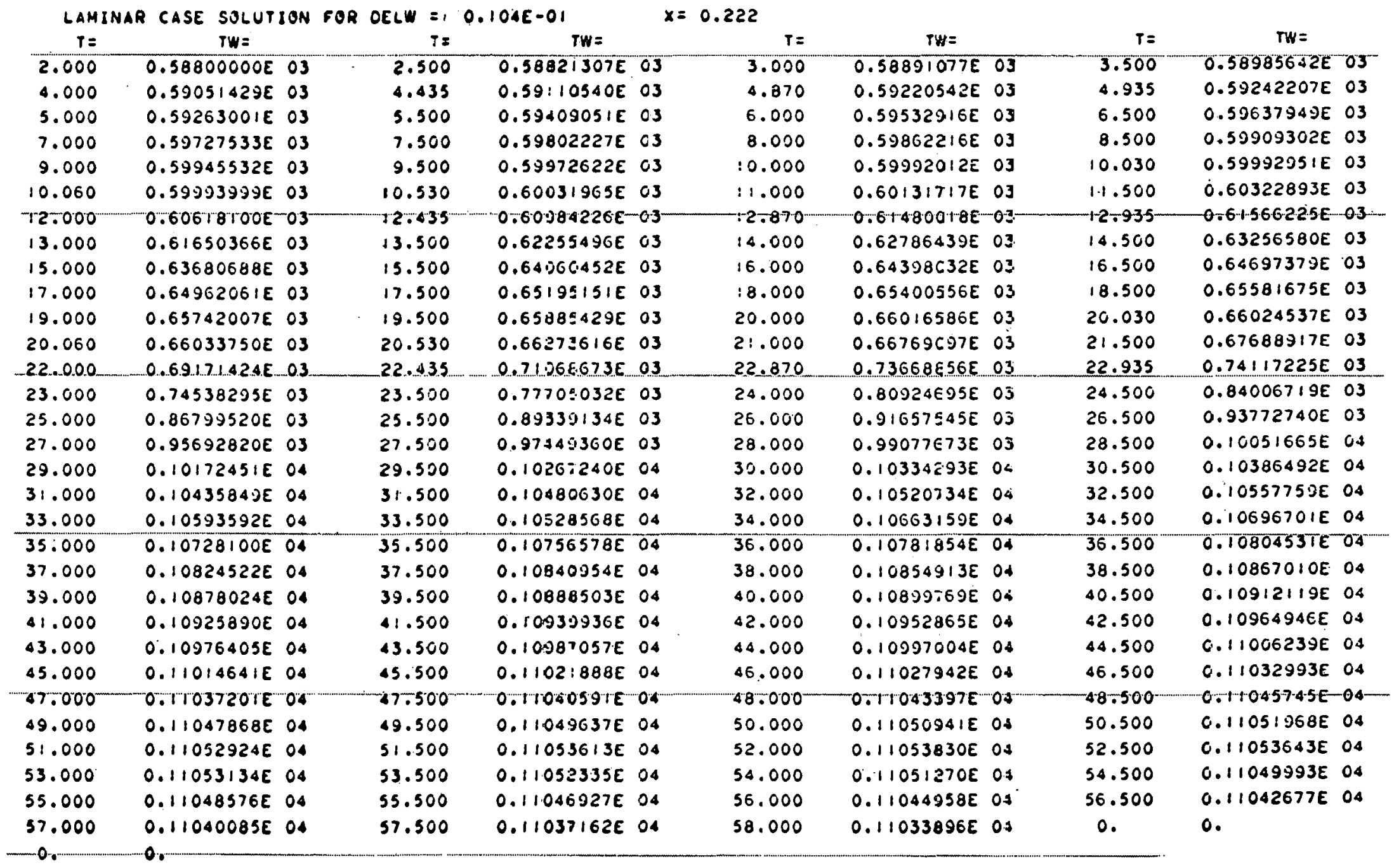


$10^{\circ}$ Cone, $x=14$ in.

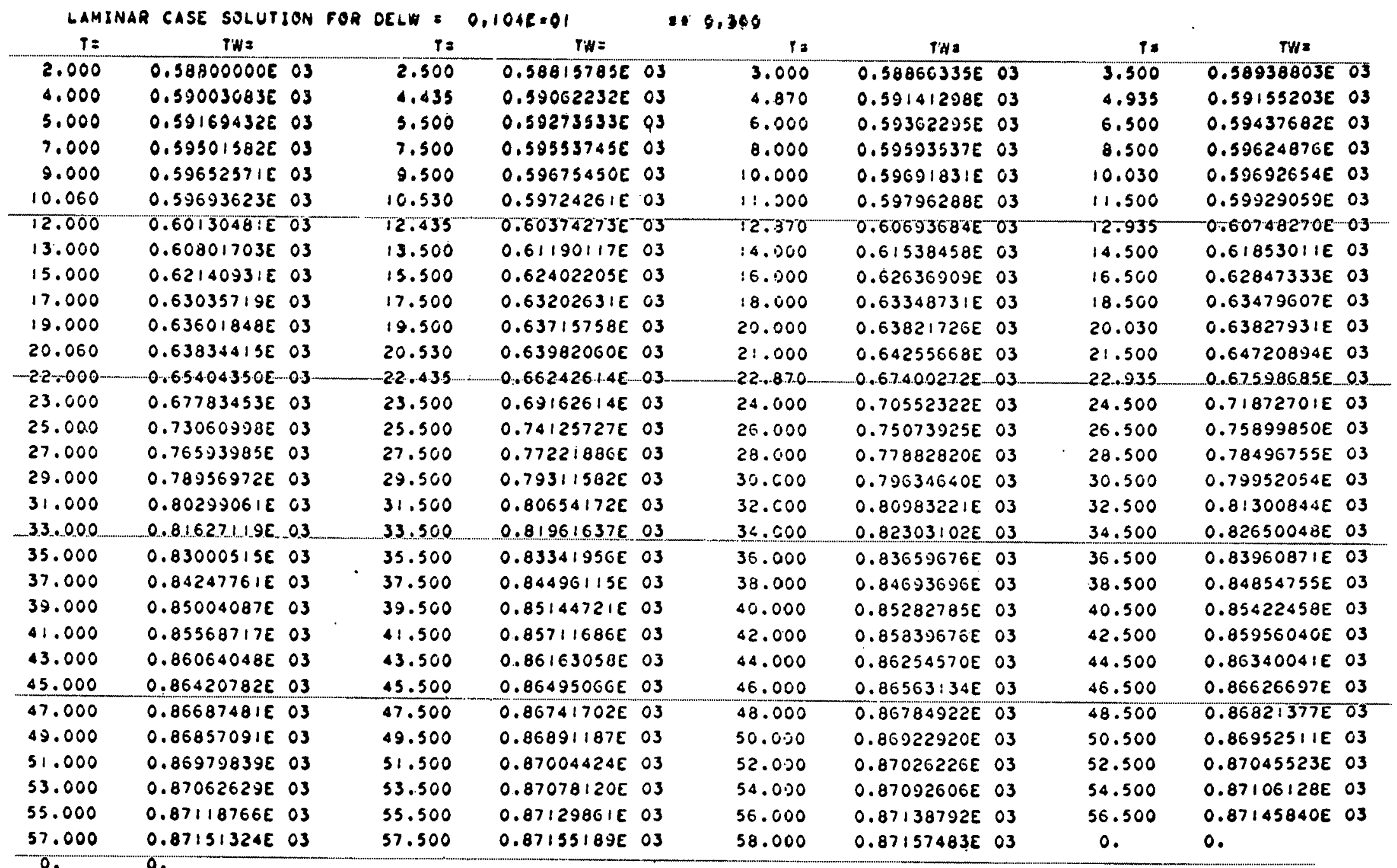




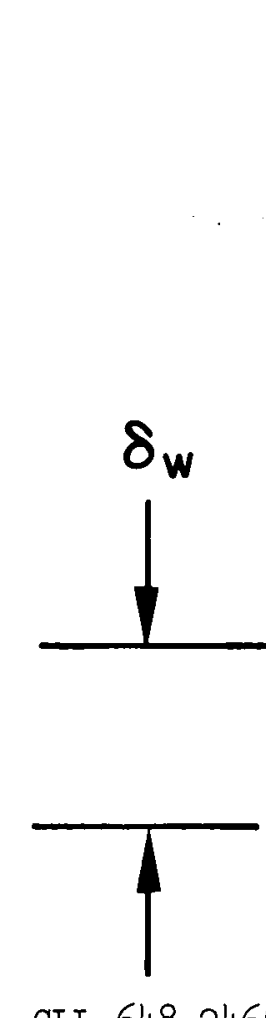

DIRECTION OF FLUID FLOW

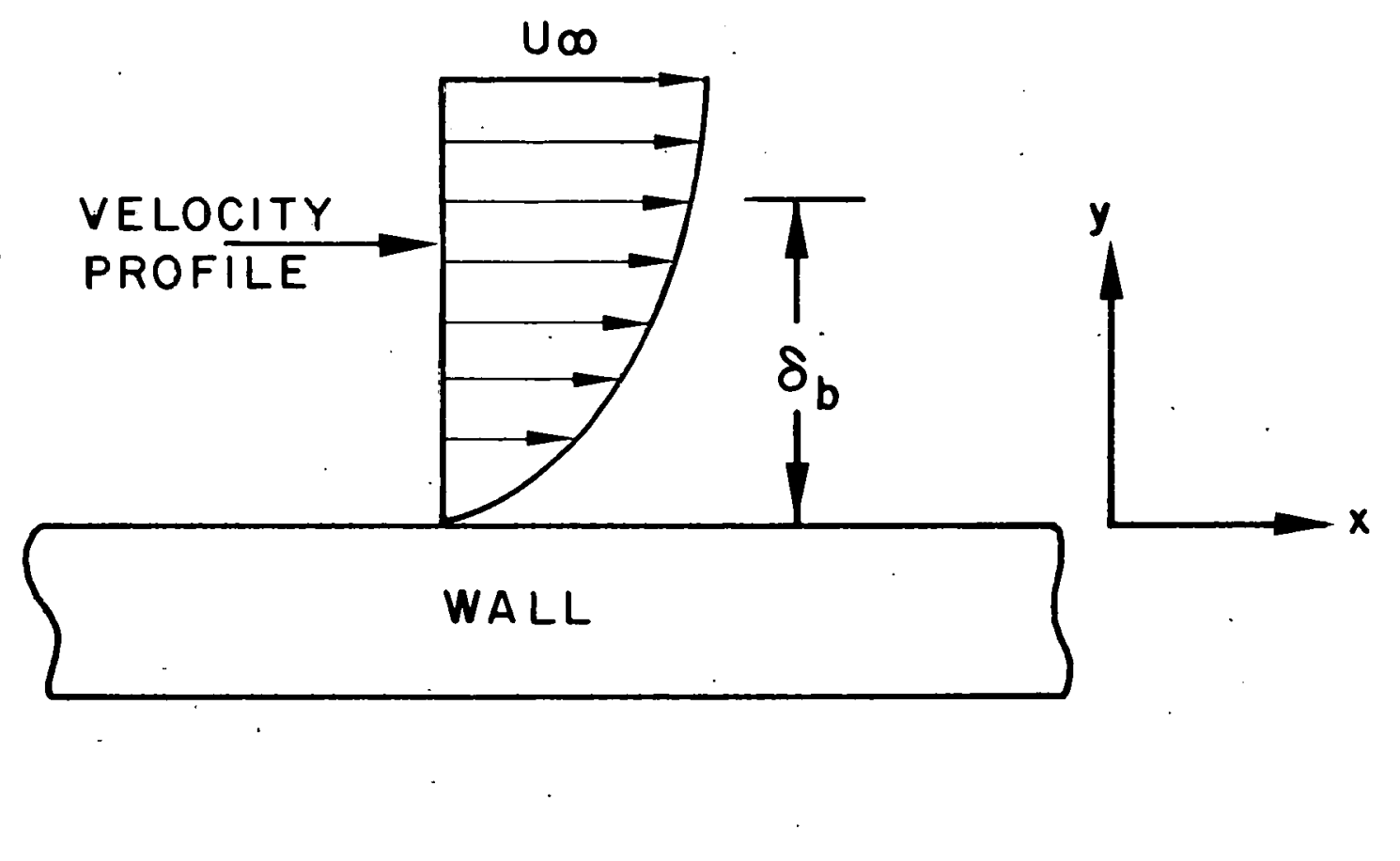

Fig. 1. Velocity profile for fluid flow along a wall. 


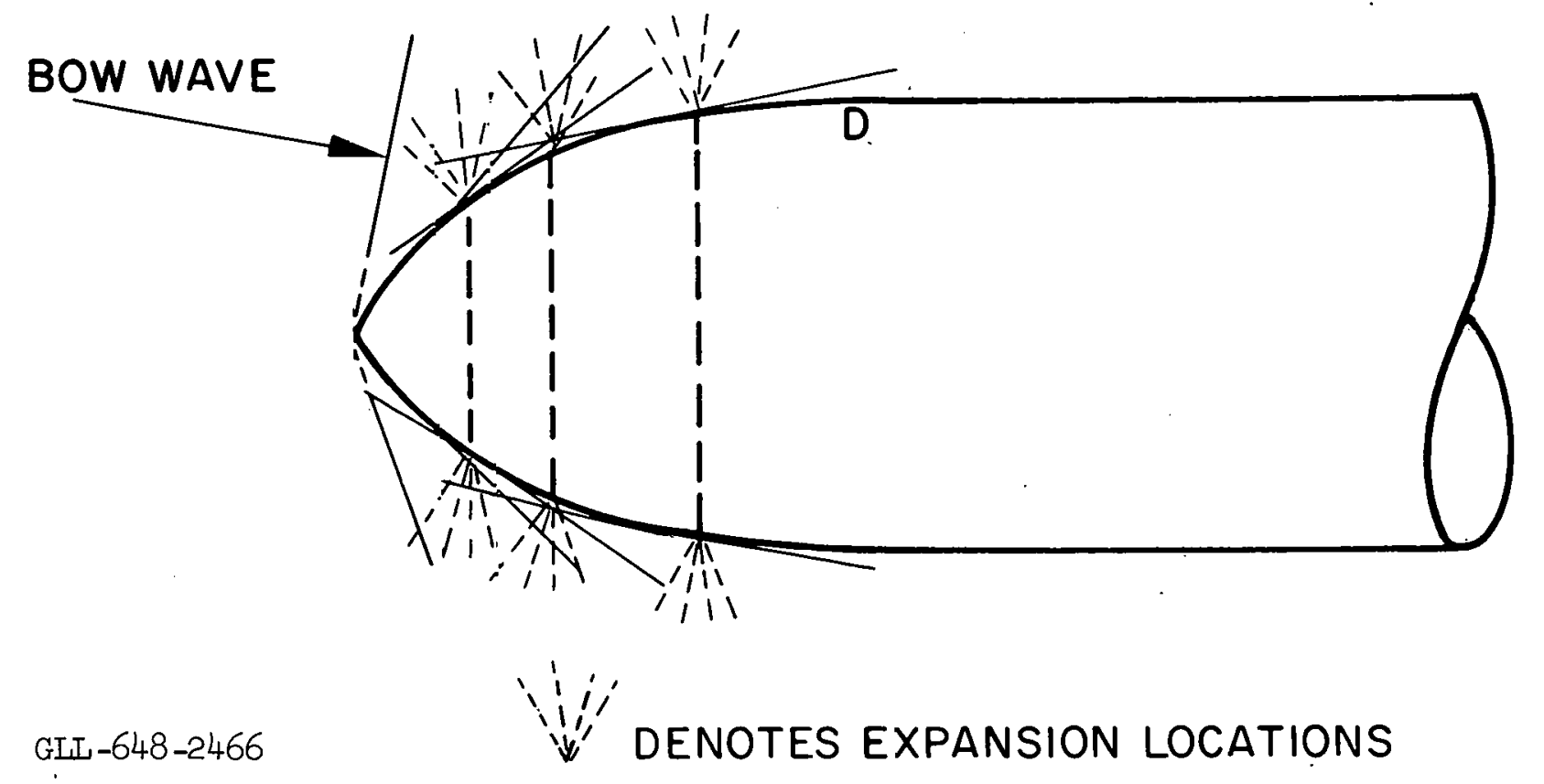

Fig. 2. Nose cone approximated by three conical segments. 


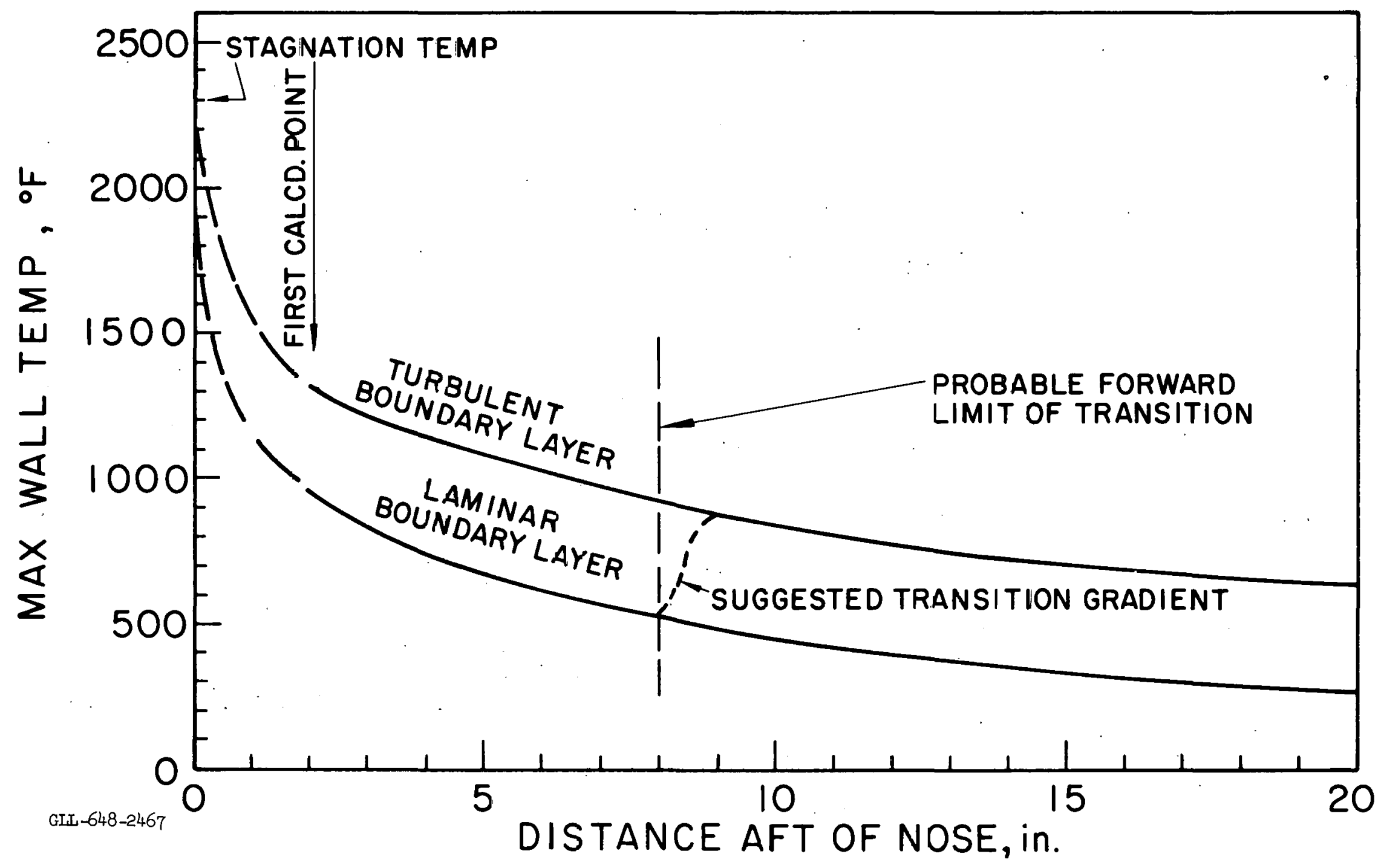

Fig. 3. Temperature distribution along HAD nose cone. Wall thickness $1 / 8$ in., time 29 sec after launch. 


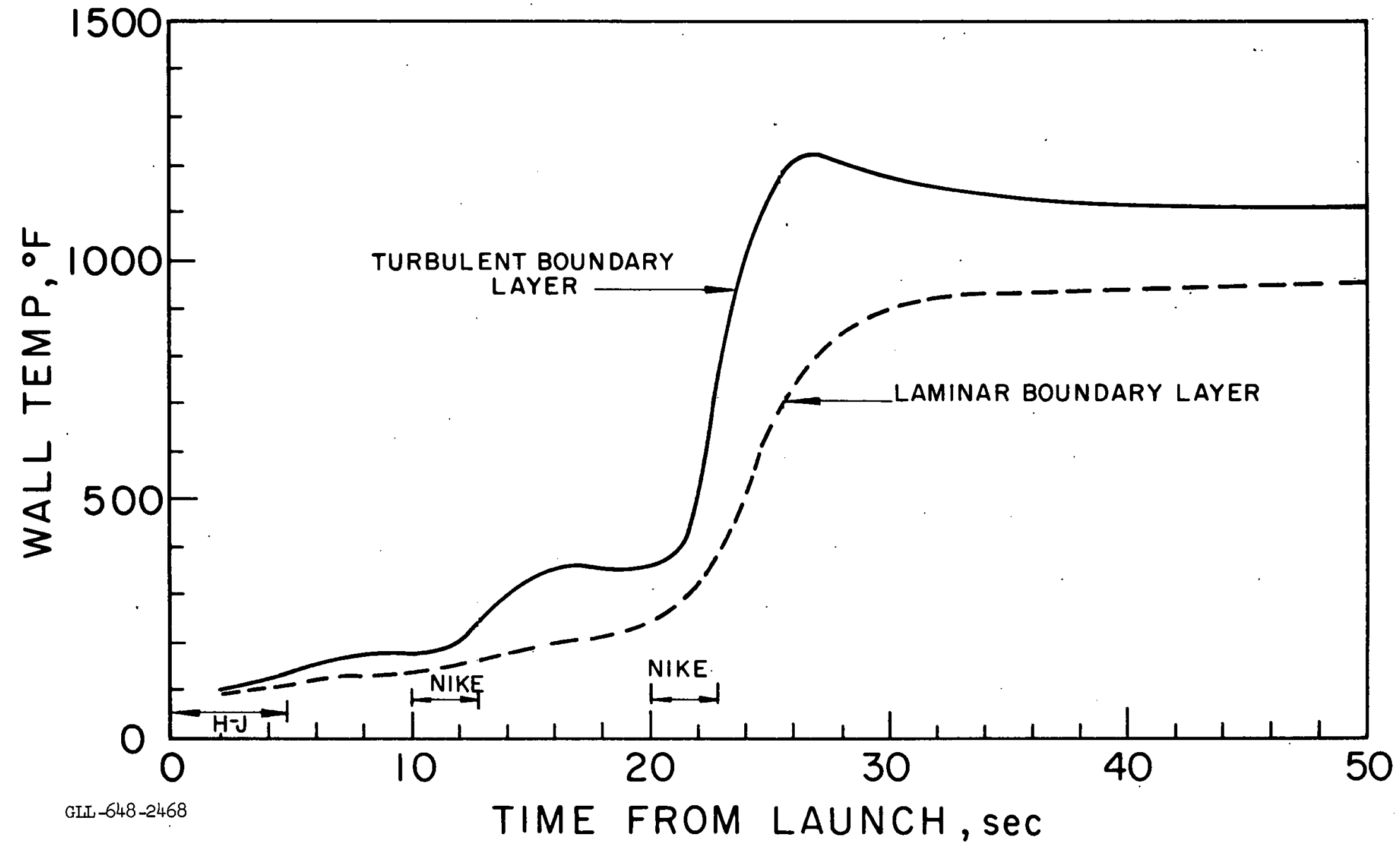

Fig. 4. Wall temperature 6 in. aft of nose as a function of time after launch. Wall thickness $1 / 8$ in. 
This report was prepared as an account of Government sponsored work. Neither the United States, nor the Commission, nor any person acting on behalf of the Commission:

A. Makes any warranty or representation, expressed or implied, with respect to the accuracy, completeness, or usefulness of the information contained in this report, or that the use of any information, apparatus, method, or process disclosed in this report may not infringe privately owned rights; or

B. Assumes any liabilities with respect to the use of, or for damages resulting from the use of any information, apparatus, method or process disclosed in this report.

As used in the above, "person acting on behalf of the Commission " includes any employee or contractor of the commission, or employee of such contractor, to the extent that such employee or contractor of the Commission, or employee of such contractor prepares, disseminates, or provides access to, any information pursuant to his employment or contract with the Commission, or his employment with such coritractor. 